\title{
Mean and uniform convergence of Lagrange interpolation with the Erdős-type weights
}

Hee Sun Jung ${ }^{1 *}$ and Ryozi Sakai ${ }^{2}$

\section{"Correspondence:}

hsun90@skku.edu

1Department of Mathematics

Education, Sungkyunkwan

University, Seoul, 110-745, Republic of Korea

Full list of author information is

available at the end of the article

\begin{abstract}
Let $\mathbb{R}=(-\infty, \infty)$, and let $Q \in C^{1}(\mathbb{R}): \mathbb{R} \rightarrow \mathbb{R}^{+}:=[0, \infty)$ be an even function. We consider the exponential-type weights $w(x)=e^{-Q(x)}, x \in \mathbb{R}$. In this paper, we obtain a mean and uniform convergence theorem for the Lagrange interpolation polynomials $L_{n}(f)$ in $L_{p}, 1<p \leq \infty$ with the weight $w$.

MSC: $41 \mathrm{~A} 05$
\end{abstract}

Keywords: exponential-type weight; Lagrange interpolation polynomial

\section{Introduction and preliminaries}

Let $\mathbb{R}=(-\infty, \infty)$, and let $Q \in C^{1}(\mathbb{R}): \mathbb{R} \rightarrow \mathbb{R}^{+}:=[0, \infty)$ be an even function, and $w(x)=$ $\exp (-Q(x))$ be the weight such that $\int_{0}^{\infty} x^{n} w^{2}(x) d x<\infty$ for all $n=0,1,2, \ldots$. Then we can construct the orthonormal polynomials $p_{n}(x)=p_{n}\left(w^{2} ; x\right)$ of degree $n$ with respect to $w^{2}(x)$. That is,

$$
\int_{-\infty}^{\infty} p_{n}(x) p_{m}(x) w^{2}(x) d x=\delta_{m n} \quad \text { (Kronecker's delta) }
$$

and

$$
p_{n}(x)=\gamma_{n} x^{n}+\cdots, \quad \gamma_{n}>0
$$

We denote the zeros of $p_{n}(x)$ by

$$
-\infty<x_{n, n}<x_{n-1, n}<\cdots<x_{2, n}<x_{1, n}<\infty
$$

We denote the Lagrange interpolation polynomial $L_{n}(f ; x)$ based at the zeros $\left\{x_{k, n}\right\}_{k=1}^{n}$ as follows:

$$
L_{n}(f ; x):=\sum_{k=1}^{n} f\left(x_{k, n}\right) l_{k, n}(x), \quad l_{k, n}(x):=\frac{p_{n}(x)}{\left(x-x_{k, n}\right) p_{n}^{\prime}\left(x_{k, n}\right)}
$$

A function $f: \mathbb{R}^{+} \rightarrow \mathbb{R}^{+}$is said to be quasi-increasing if there exists $C>0$ such that $f(x) \leq$ $C f(y)$ for $0<x<y$.

We are interested in the following subclass of weights from [1].

o 2012 Jung and Sakai; licensee Springer. This is an Open Access article distributed under the terms of the Creative Commons Attribution License (http://creativecommons.org/licenses/by/2.0), which permits unrestricted use, distribution, and reproduction in any medium, provided the original work is properly cited. 
Definition 1.1 Let $Q: \mathbb{R} \rightarrow \mathbb{R}^{+}$be an even function satisfying the following properties:

(a) $Q^{\prime}(x)$ is continuous in $\mathbb{R}$, with $Q(0)=0$.

(b) $Q^{\prime \prime}(x)$ exists and is positive in $\mathbb{R} \backslash\{0\}$.

(c) $\lim _{x \rightarrow \infty} Q(x)=\infty$.

(d) The function

$$
T(x):=\frac{x Q^{\prime}(x)}{Q(x)}, \quad x \neq 0
$$

is quasi-increasing in $(0, \infty)$ with

$$
T(x) \geq \Lambda>1, \quad x \in \mathbb{R}^{+} \backslash\{0\} .
$$

(e) There exists $C_{1}>0$ such that

$$
\frac{Q^{\prime \prime}(x)}{\left|Q^{\prime}(x)\right|} \leq C_{1} \frac{\left|Q^{\prime}(x)\right|}{Q(x)}, \quad \text { a.e. } x \in \mathbb{R} \backslash\{0\} .
$$

Then we write $w(x)=\exp (-Q(x)) \in \mathcal{F}\left(C^{2}\right)$. If there also exist a compact subinterval $J(\ni 0)$ of $\mathbb{R}$ and $C_{2}>0$ such that

$$
\frac{Q^{\prime \prime}(x)}{\left|Q^{\prime}(x)\right|} \geq C_{2} \frac{\left|Q^{\prime}(x)\right|}{Q(x)}, \quad \text { a.e. } x \in \mathbb{R} V
$$

then we write $w(x)=\exp (-Q(x)) \in \mathcal{F}\left(C^{2}+\right)$.

Example 1.2 (1) If $T(x)$ is bounded, then the weight $w=\exp (-Q)$ is called the Freud-type weight. The following example is the Freud-type weight:

$$
Q(x)=|x|^{\alpha}, \quad \alpha>1 .
$$

If $T(x)$ is unbounded, then the weight $w=\exp (-Q)$ is called the Erdös-type weight. The following examples give the Erdős-type weights $w=\exp (-Q)$.

(2) [2, Theorem 3.1] For $\alpha>1, l=1,2,3, \ldots$

$$
Q(x)=Q_{l, \alpha}(x)=\exp _{l}\left(|x|^{\alpha}\right)-\exp _{l}(0)
$$

where

$$
\exp _{l}(x)=\exp (\exp (\exp \cdots \exp x) \cdots) \quad(l \text {-times })
$$

More generally, we define for $\alpha+u>1, \alpha \geq 0, u \geq 0$ and $l \geq 1$,

$$
Q_{l, \alpha, u}(x):=|x|^{u}\left(\exp _{l}\left(|x|^{\alpha}\right)-\alpha^{*} \exp _{l}(0)\right)
$$

where $\alpha^{*}=0$ if $\alpha=0$, otherwise $\alpha^{*}=1$. (We note that $Q_{l, 0, u}(x)$ gives a Freud-type weight.)

(3) We define $Q_{\alpha}(x):=(1+|x|)^{|x|^{\alpha}}-1, \alpha>1$. 
In this paper, we investigate the convergence of the Lagrange interpolation polynomials with respect to the weight $w \in \mathcal{F}\left(C^{2}+\right)$. When we consider the Erdős-type weights, the following definition follows from Damelin and Lubinsky [3].

Definition 1.3 Let $w(x)=\exp (-Q(x))$, where $Q: \mathbb{R} \rightarrow \mathbb{R}$ is even and continuous. $Q^{\prime \prime}$ exists in $(0, \infty), Q^{(j)} \geq 0$, in $(0, \infty), j=0,1,2$, and the function

$$
T^{*}(x):=1+\frac{x Q^{\prime \prime}(x)}{Q^{\prime}(x)}
$$

is increasing in $(0, \infty)$ with

$$
\lim _{x \rightarrow \infty} T^{*}(x)=\infty ; \quad T^{*}(0+):=\lim _{x \rightarrow 0+} T^{*}(x)>1
$$

Moreover, we assume that for some constants $C_{1}, C_{2}, C_{3}>0$,

$$
C_{1} \leq T^{*}(x) /\left(\frac{x Q^{\prime}(x)}{Q(x)}\right) \leq C_{2}, \quad x \geq C_{3},
$$

and for every $\varepsilon>0$,

$$
T^{*}(x)=O\left(Q(x)^{\varepsilon}\right), \quad x \rightarrow \infty .
$$

Then we write $w \in \mathcal{E}$.

Damelin and Lubinsky [3] got the following results with the Erdős-type weights $w=$ $\exp (-Q) \in \mathcal{E}$.

Theorem A $\left(\left[3\right.\right.$, Theorem 1.3]) Let $w=\exp (-Q) \in \mathcal{E}$. Let $L_{n}(f, x)$ denote the Lagrange interpolation polynomial to $f$ at the zeros of $p_{n}\left(w^{2}, x\right)$. Let $1<p<\infty, \Delta \in \mathbb{R}, \kappa>0$. Then for

$$
\lim _{n \rightarrow \infty}\left\|\left(f-L_{n}(f)\right) w(1+Q)^{-\Delta}\right\|_{L_{p}(\mathbb{R})}=0
$$

to hold for every continuous function $f: \mathbb{R} \rightarrow \mathbb{R}$ satisfying

$$
\lim _{|x| \rightarrow \infty}\left|f(x) w(x)(\log |x|)^{1+\kappa}\right|=0
$$

it is necessary and sufficient that

$$
\Delta>\max \left\{0, \frac{2}{3}\left(\frac{1}{4}-\frac{1}{p}\right)\right\} \text {. }
$$

Our main purpose in this paper is to give mean and uniform convergence theorems with respect to $\left\{L_{n}(f)\right\}, n=1,2, \ldots$, in $L_{p}$-norm, $1<p \leq \infty$. The proof for $1<p<\infty$ will be shown by use of the method of Damelin and Lubinsky. In Section 2, we write the main theorems. In Section 3, we prepare some fundamental lemmas; and in Section 4, we will prove the theorem for $1<p<\infty$. Finally, we will prove the theorem for the uniform convergence in Section 5. 
For any nonzero real-valued functions $f(x)$ and $g(x)$, we write $f(x) \sim g(x)$ if there exist constants $C_{1}, C_{2}>0$ independent of $x$ such that $C_{1} g(x) \leq f(x) \leq C_{2} g(x)$ for all $x$. Similarly, for any two sequences of positive numbers $\left\{c_{n}\right\}_{n=1}^{\infty}$ and $\left\{d_{n}\right\}_{=1}^{\infty}$, we define $c_{n} \sim d_{n}$. We denote the class of polynomials of degree at most $n$ by $\mathcal{P}_{n}$.

Throughout $C, C_{1}, C_{2}, \ldots$ denote positive constants independent of $n, x, t$, and polynomials of degree at most $n$. The same symbol does not necessarily denote the same constant in different occurrences.

\section{Theorems}

In the following, we introduce useful notations. Mhaskar-Rakhmanov-Saff numbers (MRS) $a_{x}$ are defined as the positive roots of the following equations:

$$
x=\frac{2}{\pi} \int_{0}^{1} \frac{a_{x} u Q^{\prime}\left(a_{x} u\right)}{\left(1-u^{2}\right)^{\frac{1}{2}}} d u, \quad x>0 .
$$

The function $\varphi_{u}(x)$ is defined as follows:

$$
\varphi_{u}(x)= \begin{cases}\frac{a_{u} \frac{1-\frac{|x|}{a_{u}}}{u} \frac{|x|}{\sqrt{1-\frac{|x|}{a_{u}}}+\delta_{u}},}{} & |x| \leq a_{u}, \\ \varphi_{u}\left(a_{u}\right), & a_{u}<|x|,\end{cases}
$$

where

$$
\delta_{x}=\left(x T\left(a_{x}\right)\right)^{-\frac{2}{3}}, \quad x>0 .
$$

We define

$$
\Phi(x):=\frac{1}{(1+Q(x))^{\frac{2}{3}} T(x)}
$$

and

$$
\Phi_{n}(x):=\max \left\{\delta_{n}, 1-\frac{|x|}{a_{n}}\right\} .
$$

Here we note that for $0<d \leq|x|$,

$$
\Phi(x) \sim \frac{Q(x)^{\frac{1}{3}}}{x Q^{\prime}(x)}
$$

and we see

$$
\Phi(x) \leq C \Phi_{n}(x), \quad n \geq 1
$$

(see Lemma 3.3 below). Moreover, we define

$$
\Phi^{\left(\frac{1}{4}-\frac{1}{p}\right)^{+}}(x):= \begin{cases}1, & 0<p<4, \\ \Phi^{\frac{1}{4}-\frac{1}{p}}(x), & 4 \leq p \leq \infty .\end{cases}
$$


Let $1<p<\infty$. We give a convergence theorem as an analogy of Theorem $\mathrm{A}$ for $L_{n}(f)$ in $L_{p}$-norm. We need to prepare a lemma.

Lemma 2.1 ([4, Theorem 1.6]) Let $w=\exp (-Q) \in \mathcal{F}\left(C^{2}+\right)$.

(a) Let $T(x)$ be unbounded. Then for any $\eta>0$, there exists a constant $C(\eta)>0$ such that for $t \geq 1$,

$$
a_{t} \leq C(\eta) t^{\eta}
$$

(b) Assume

$$
\frac{Q^{\prime \prime}(x)}{Q^{\prime}(x)} \leq \lambda(b) \frac{Q^{\prime}(x)}{Q(x)}, \quad|x| \geq b>0,
$$

where $b>0$ is large enough. Suppose that there exist constants $\eta>0$ and $C_{1}>0$ such that $a_{t} \leq C_{1} t^{\eta}$. If $\lambda:=\lambda(b)>1$, then there exists a constant $C(\lambda, \eta)$ such that for $a_{t} \geq 1$,

$$
T\left(a_{t}\right) \leq C(\lambda, \eta) t^{\frac{2(\eta+\lambda-1)}{\lambda+1}} .
$$

If $0<\lambda \leq 1$, then for any $\mu>0$, there exists $C(\lambda, \mu)$ such that

$$
T\left(a_{t}\right) \leq C(\lambda, \mu) t^{\mu}, \quad t \geq 1
$$

For a fixed constant $\beta>0$, we define

$$
\phi(x):=\left(1+x^{2}\right)^{-\beta / 2} .
$$

Using this function, we have the following theorem. We suppose that the weight $w$ is the Erdős-type weight.

Our theorem is as follows. Let $f \in C_{0}(\mathbb{R})$ mean that $f \in C(\mathbb{R})$ and $\lim _{|x| \rightarrow \infty} f(x)=0$.

Theorem 2.2 Let $w=\exp (-Q) \in \mathcal{F}\left(C^{2}+\right)$, and let $T(x)$ be unbounded. Let $1<p<\infty$ and $\beta>0$, and let us define $\phi$ as (2.4), and $\lambda=\lambda(b) \geq 1$ as (2.1). We suppose that for $f \in C(\mathbb{R})$,

$$
\phi^{-1}(x) w(x) f(x) \in C_{0}(\mathbb{R})
$$

and

$$
\Delta>\frac{9}{4} \frac{\lambda-1}{3 \lambda-1} .
$$

Then we have

$$
\lim _{n \rightarrow \infty}\left\|\left(f-L_{n}(f)\right) w \Phi^{\Delta+\left(\frac{1}{4}-\frac{1}{p}\right)^{+}}\right\|_{L_{p}(\mathbb{R})}=0 .
$$

We remark that if $w \in \mathcal{F}\left(C^{2}+\right)$ is the Erdös-type weight, then we have $\lambda=\lambda(b) \geq 1$ in (2.1). In fact, if $\lambda<1$, then by Lemma 3.9 below, we see that for $x \geq b>0$,

$$
T(x)=\frac{x Q^{\prime}(x)}{Q(x)} \leq \frac{x}{Q(x)} Q^{\prime}(b)\left(\frac{Q(x)}{Q(b)}\right)^{\lambda}=\frac{Q^{\prime}(b)}{Q(b)^{\lambda}} \frac{x}{Q(x)^{1-\lambda}} \rightarrow 0 \quad \text { as } x \rightarrow \infty .
$$


This contradicts our assumption for $T(x)$. In Example 1.2, we consider the weight $w_{l, \alpha, m}=$ $\exp \left(-Q_{l, \alpha, m}\right)$. In (2.1), we set $Q:=Q_{l, \alpha, m}$ and $\lambda:=\lambda(b)$. If $w_{l, \alpha, m}$ is an Erdős-type weight, that is, $T(x):=T_{l, \alpha, m}(x)$ is unbounded, then it is easy to show

$$
\lim _{b \rightarrow \infty} \lambda(b)=1
$$

Therefore, when we give any $\Delta>0$, there exists a constant $b$ large enough such that

$$
\Delta>\frac{9}{4} \frac{\lambda(b)-1}{3 \lambda(b)-1}
$$

Hence, we have the following corollary.

Corollary 2.3 Let $1<p<\infty$ and $\Delta>0$. Then for the weight $w_{l, \alpha, m}=\exp \left(-Q_{l, \alpha, m}\right)(\alpha>0)$, we have

$$
\lim _{n \rightarrow \infty}\left\|\left(f-L_{n}(f)\right) w_{l, \alpha, m} \Phi^{\Delta+\left(\frac{1}{4}-\frac{1}{p}\right)^{+}}\right\|_{L_{p}(\mathbb{R})}=0
$$

We also consider the case of $p=\infty$.

Theorem 2.4 Let $w=\exp (-Q) \in \mathcal{F}\left(C^{2}+\right)$, and let $T(x)$ be unbounded. For every $f \in C_{0}(\mathbb{R})$ and $n \geq 1$, we have

$$
\left\|\left(f-L_{n}(f)\right) w \Phi^{3 / 4}\right\|_{L_{\infty}(\mathbb{R})} \leq C E_{n-1}(w ; f) \log n
$$

where

$$
E_{m}(w ; f)=\inf _{P_{m} \in \mathcal{P}_{m}} \max _{x \in \mathbb{R}}\left|\left(f(x)-P_{m}(x)\right) w(x)\right|, \quad m=0,1,2, \ldots
$$

Moreover, if $f^{(r)}, r \geq 1$, is an integer, then for $n>r+1$ we have

$$
\left\|\left(f-L_{n}(f)\right) w \Phi^{3 / 4}\right\|_{L_{\infty}(\mathbb{R})} \leq C\left(\frac{a_{n}}{n}\right)^{r} E_{n-r-1}\left(w ; f^{(r)}\right) \log n .
$$

\section{Fundamental lemmas}

To prove the theorems we need some lemmas.

Lemma 3.1 Let $w=\exp (-Q) \in \mathcal{F}\left(C^{2}+\right)$. Then we have the following.

(a) [1, Lemma 3.11(a), (b)] Given fixed $0<\alpha, \alpha \neq 1$, we have uniformly for $t>0$,

$$
\left|1-\frac{a_{\alpha t}}{a_{t}}\right| \sim \frac{1}{T\left(a_{t}\right)}
$$

and we have for $t>0$,

$$
\left|1-\frac{a_{t}}{a_{s}}\right| \sim \frac{1}{T\left(a_{t}\right)}\left|1-\frac{t}{s}\right|, \quad \frac{1}{2} \leq \frac{t}{s} \leq 2 .
$$

(b) $[1$, Lemma 3.7 (3.38)] For some $0<\varepsilon \leq 2$, and for large enough $t$,

$$
T\left(a_{t}\right) \leq t^{2-\varepsilon} .
$$


Lemma 3.2 Let $w=\exp (-Q) \in \mathcal{F}\left(C^{2}+\right)$. Then we have the following.

(a) [1, Lemma 3.5(a), (b)] Let $L>0$ be a fixed constant. Uniformly for $t>0$,

$$
Q\left(a_{L t}\right) \sim Q\left(a_{t}\right) \text { and } Q^{\prime}\left(a_{L t}\right) \sim Q^{\prime}\left(a_{t}\right) .
$$

Moreover,

$$
a_{L t} \sim a_{t} \text { and } T\left(a_{L t}\right) \sim T\left(a_{t}\right) .
$$

(b) [1, Lemma 3.4 (3.18), (3.17)] Uniformly for $x>0$ with $a_{t}:=x, t>0$, we have

$$
Q^{\prime}(x) \sim \frac{t \sqrt{T(x)}}{a_{t}} \text { and } Q(x) \sim \frac{t}{\sqrt{T(x)}} .
$$

(c) $\left[1\right.$, Lemma 3.8(a)] For $x \in\left[0, a_{t}\right)$,

$$
Q^{\prime}(x) \leq C \frac{t}{a_{t}} \frac{1}{\sqrt{1-\frac{x}{a_{t}}}} .
$$

Lemma 3.3 Let $w=\exp (-Q) \in \mathcal{F}\left(C^{2}+\right)$. For $x \in \mathbb{R}$, we have

$$
\Phi(x) \leq C \Phi_{n}(x), \quad n \geq 1 .
$$

Proof Let $x=a_{u}, u \geq 1$. By Lemma 3.2(b), we have

$$
u \sim Q\left(a_{u}\right) \sqrt{T\left(a_{u}\right)}
$$

So, we have

$$
\delta_{u}^{-1} \sim Q^{\frac{2}{3}}\left(a_{u}\right) T\left(a_{u}\right)=\frac{a_{u} Q^{\prime}\left(a_{u}\right)}{Q^{\frac{1}{3}}\left(a_{u}\right)}=\frac{x Q^{\prime}(x)}{Q^{\frac{1}{3}}(x)} .
$$

Now, if $u \leq \frac{n}{2}$, then we have

$$
\begin{aligned}
1-\frac{a_{u}}{a_{n}} & \geq 1-\frac{a_{n / 2}}{a_{n}} \sim \frac{1}{T\left(a_{n}\right)} \quad(\text { by Lemma 3.1(a)) } \\
& \geq \frac{1}{\left(n T\left(a_{n}\right)\right)^{\frac{2}{3}}}=\delta_{n} \quad(\text { by Lemma 3.1(b)). }
\end{aligned}
$$

So, we have

$$
\begin{aligned}
\Phi_{n}(x) & =1-\frac{a_{u}}{a_{n}} \geq 1-\frac{a_{u}}{a_{2 u}} \sim \frac{1}{T\left(a_{u}\right)} \quad(\text { by Lemma 3.1(a)) } \\
& \geq \frac{1}{\left(u T\left(a_{u}\right)\right)^{\frac{2}{3}}}=\delta_{u} \sim \Phi(x) \quad(\text { by Lemma 3.2(b) and (3.1)). }
\end{aligned}
$$

Let $\frac{n}{2}<u<n$. Then we have

$$
\Phi_{n}(x) \geq \delta_{n} \sim \delta_{u} \sim \Phi(x) \quad \text { (by Lemma 3.2(a), (b) and (3.1)). }
$$


Lemma 3.4 Let $w \in \mathcal{F}\left(C^{2}+\right)$. Then we have the following.

(a) $[1$, Theorem $1.19(\mathrm{f})]$ For the minimum positive zero $x_{[n / 2], n}$,

$$
x_{[n / 2], n} \sim \frac{a_{n}}{n},
$$

and for the maximum zero $x_{1, n}$,

$$
1-\frac{x_{1, n}}{a_{n}} \sim \delta_{n}
$$

(b) [1, Theorem 1.19(e)] For $n \geq 1$ and $1 \leq j \leq n-1$,

$$
x_{j, n}-x_{j+1, n} \sim \varphi_{n}\left(x_{j, n}\right) .
$$

(c) [1, p.329, (12.20)] Uniformly for $n \geq 1,1 \leq k \leq n-1$,

$$
\varphi_{n}\left(x_{k, n}\right) \sim \varphi_{n}\left(x_{k+1, n}\right) .
$$

(d) Let $\max \left\{\left|x_{k, n}\right|,\left|x_{k+1, n}\right|\right\} \leq a_{\alpha n}, 0<\alpha<1$. Then we have

$$
w\left(x_{k, n}\right) \sim w\left(x_{k+1, n}\right) \sim w(x) \quad\left(x_{k+1, n} \leq x \leq x_{k, n}\right) .
$$

So, for given $C>0$ and $|x| \leq a_{\beta n}, 0<\beta<\alpha$, if $\left|x-x_{k, n}\right| \leq C \varphi_{n}(x)$, then we have

$$
w(x) \sim w\left(x_{k, n}\right) .
$$

Proof (d) Let $\max \left\{\left|x_{k, n}\right|,\left|x_{k+1, n}\right|\right\}=\left|x_{k, n}\right|$ (for the case of $\max \left\{\left|x_{k, n}\right|,\left|x_{k+1, n}\right|\right\}=\left|x_{k+1, n}\right|$, we also have the result similarly). By (b) there exists a constant $C>0$ such that

$$
\left|x_{k, n}-x_{k+1, n}\right| \leq C \varphi_{n}\left(x_{k, n}\right)
$$

Then we see

$$
\begin{aligned}
\varphi_{n}\left(x_{k, n}\right) & \sim \frac{a_{n}}{n} \frac{1-\frac{\left|x_{k, n}\right|}{a_{2 n}}}{\sqrt{1-\frac{\left|x_{k, n}\right|}{a_{n}}}}=\frac{a_{n}}{n} \frac{1-\frac{\left|x_{k, n}\right|}{a_{n}}+\left|x_{k, n}\right|\left\{\frac{1}{a_{n}}-\frac{1}{a_{2 n}}\right\}}{\sqrt{1-\frac{\left|x_{k, n}\right|}{a_{n}}}} \\
& =\frac{a_{n}}{n} \frac{1-\frac{\left|x_{k, n}\right|}{a_{n}}+\frac{\left|x_{k, n}\right|}{a_{n}}\left(1-\frac{a_{n}}{a_{2 n}}\right)}{\sqrt{1-\frac{\left|x_{k, n}\right|}{a_{n}}}} \sim \frac{a_{n}}{n} \frac{1-\frac{\left|x_{k, n}\right|}{a_{n}}+C \frac{\left|x_{k, n}\right|}{a_{n}} \frac{1}{T\left(a_{n}\right)}}{\sqrt{1-\frac{\left|x_{k, n}\right|}{a_{n}}}} \\
& \sim \frac{a_{n}}{n} \sqrt{1-\frac{\left|x_{k, n}\right|}{a_{n}}} .
\end{aligned}
$$

Therefore, from (3.2) and Lemma 3.2(c), we have

$$
\begin{aligned}
\left|Q\left(x_{k, n}\right)-Q\left(x_{k+1, n}\right)\right| & =\left|Q^{\prime}(\xi)\left(x_{k, n}-x_{k+1, n}\right)\right| \leq C\left|Q^{\prime}(\xi)\right| \varphi_{n}(x) \quad\left(x_{k+1, n} \leq \xi \leq x_{k, n}\right) \\
& \leq C\left|Q^{\prime}\left(x_{k, n}\right)\right| \frac{a_{n}}{n} \sqrt{1-\frac{\left|x_{k, n}\right|}{a_{n}}} \leq C \frac{n}{a_{n}} \frac{1}{\sqrt{1-\frac{\left|x_{k, n}\right|}{a_{n}}}} \frac{a_{n}}{n} \sqrt{1-\frac{\left|x_{k, n}\right|}{a_{n}}} \leq C .
\end{aligned}
$$




\section{Consequently,}

$$
w\left(x_{k, n}\right) \sim w\left(x_{k+1, n}\right) \sim w(x) \quad\left(x_{k+1, n} \leq x \leq x_{k, n}\right) .
$$

Let $\left|x-x_{k, n}\right| \leq C \varphi_{n}(x)$ and $|x| \leq a_{\beta n}$. Then we see that there exists $n_{0}>0$ such that $\left|x_{k, n}\right| \leq$ $a_{\alpha n}, n \geq n_{0}$. In fact, we can show it as follows. We use Lemma 3.1(a) and (b). For $|x| \leq a_{\beta n}$, we see

$$
\left|x_{k, n}\right| \leq|x|+C \varphi_{n}(x) \leq|x|+C \frac{a_{n}}{n} \sqrt{1-\frac{|x|}{a_{n}}}
$$

and if we take $n$ large enough, then we have

$$
\begin{aligned}
\frac{d}{d t}\left(t+C \frac{a_{n}}{n} \sqrt{1-\frac{t}{a_{n}}}\right) & =1-C \frac{1}{n} \frac{1}{2 \sqrt{1-\frac{t}{a_{n}}}} \geq 1-C \frac{1}{n} \frac{1}{2 \sqrt{1-\frac{a_{n / 3}}{a_{n}}}} \\
& \geq 1-C \frac{\sqrt{T\left(a_{n}\right)}}{2 n} \geq 1-C \frac{1}{2 n^{\varepsilon / 2}}>0,
\end{aligned}
$$

that is, $g(t)=t+C \frac{a_{n}}{n} \sqrt{1-\frac{t}{a_{n}}}$ is increasing. So, we see

$$
\left|x_{k, n}\right| \leq a_{\beta n}+C \frac{a_{n}}{n} \sqrt{1-\frac{a_{\beta n}}{a_{n}}} \leq a_{\beta n}+C \frac{a_{n}}{n} \frac{1}{\sqrt{T\left(a_{n}\right)}} .
$$

Therefore, we have

$$
\begin{aligned}
a_{\alpha n}-\left(a_{\beta n}+C \frac{a_{n}}{n} \frac{1}{\sqrt{T\left(a_{n}\right)}}\right) & \sim \frac{a_{n}}{T\left(a_{n}\right)}-C \frac{a_{n}}{n} \frac{1}{\sqrt{T\left(a_{n}\right)}} \\
& =\frac{a_{n}}{T\left(a_{n}\right)}\left(1-C \frac{\sqrt{T\left(a_{n}\right)}}{n}\right) \geq \frac{a_{n}}{T\left(a_{n}\right)}\left(1-C \frac{1}{n^{\varepsilon / 2}}\right)>0 .
\end{aligned}
$$

Now, we can show (d). Without loss of generality, we may assume $x \in\left[x_{j+1, n}, x_{j, n}\right] \subset$ $\left\{x_{k, n}|| x-x_{k, n} \mid \leq C \varphi_{n}(x)\right\}$. We define

$$
x_{k_{1}, n}:=\min \left\{x_{k, n}|| x-x_{k, n} \mid \leq C \varphi_{n}(x)\right\}, \quad x_{k_{2}, n}:=\max \left\{x_{k, n}|| x-x_{k, n} \mid \leq C \varphi_{n}(x)\right\} .
$$

Here we note that $k_{1}, k_{2}$ are decided depending only on the constant $C$. Then by former result, we have

$$
w\left(x_{k_{1}, n}\right) \sim w\left(x_{k_{2}, n}\right) \sim w(x) \quad\left(x_{k_{1}, n} \leq x \leq x_{k_{2}, n}\right) .
$$

Lemma 3.5 Let $w=\exp (-Q) \in \mathcal{F}\left(C^{2}+\right)$. Then we have the following.

(a) [1, Theorem 1.17] Uniformly for $n \geq 1$,

$$
\sup _{x \in \mathbb{R}}\left|p_{n}(x)\right| w(x)\left|x^{2}-a_{n}^{2}\right|^{\frac{1}{4}} \sim 1 \text {. }
$$

(b) [1, Theorem 1.19(a)] Uniformly for $n \geq 1$ and $1 \leq j \leq n$,

$$
\left|\left(p_{n}^{\prime} w\right)\left(x_{j, n}\right)\right| \sim \varphi_{n}^{-1}\left(x_{j, n}\right) a_{n}^{-\frac{1}{2}}\left(1-\frac{\left|x_{j, n}\right|}{a_{n}}\right)^{-\frac{1}{4}} .
$$


(c) [1, Theorem 1.19(d)] For $x \in\left[x_{k+1, n}, x_{k, n}\right]$, if $k \leq n-1$,

$$
\left|p_{n}(x) w(x)\right| \sim \min \left\{\left|x-x_{k, n}\right|,\left|x-x_{k+1, n}\right|\right\} a_{n}^{1 / 2} \varphi_{n}(x)^{-1}\left(1-\frac{\left|x_{k, n}\right|}{a_{n}}\right)^{-1 / 4} .
$$

Lemma 3.6 (cf. [5, Theorem 2.7]) Let $w \in \mathcal{F}\left(C^{2}+\right)$ and $0<p \leq \infty$. Then uniformly $n \geq 2$,

$$
\left\|\Phi^{\left(\frac{1}{4}-\frac{1}{p}\right)^{+}} p_{n} w\right\|_{L_{p}(\mathbb{R})} \leq C a_{n}^{\frac{1}{p}-\frac{1}{2}} \begin{cases}1, & 0<p<4 \text { or } p=\infty \\ \log (1+n), & 4 \leq p,\end{cases}
$$

where $x^{+}=0$ if $x \leq 0, x^{+}=x$ if $x>0$.

Proof From Lemma 3.3, we know $\Phi(x) \leq \Phi_{n}(x)$, then in [5, Theorem 2.7] we only exchange $\Phi_{n}$ with $\Phi$.

Let $f \in L_{p, w}(\mathbb{R})$. The Fourier-type series of $f$ is defined by

$$
\tilde{f}(x):=\sum_{k=0}^{\infty} a_{k}\left(w^{2}, f\right) p_{k}\left(w^{2}, x\right), \quad a_{k}\left(w^{2}, f\right):=\int_{-\infty}^{\infty} f(t) p_{k}\left(w^{2}, t\right) w^{2}(t) d t
$$

We denote the partial sum of $\tilde{f}(x)$ by

$$
s_{n}(f, x):=s_{n}\left(w^{2}, f, x\right):=\sum_{k=0}^{n-1} a_{k}\left(w^{2}, f\right) p_{k}\left(w^{2}, x\right)
$$

The partial sum $s_{n}(f)$ admits the representation

$$
s_{n}(f, x)=\sum_{j=0}^{n-1} a_{j} p_{j}(x)=\int_{-\infty}^{\infty} f(t) K_{n}(x, t) w^{2}(t) d t
$$

where

$$
K_{n}(x, t):=\sum_{j=0}^{n-1} p_{j}(x) p_{j}(t)
$$

The Christoffel-Darboux formula

$$
K_{n}(x, t)=\frac{\gamma_{n-1}}{\gamma_{n}} \frac{p_{n}(x) p_{n-1}(t)-p_{n-1}(x) p_{n}(t)}{x-t}
$$

is well known (see [6, Theorem 1.1.4]).

Lemma 3.7 ([6, Lemma 9.2.6]) Let $1<p<\infty$ and $g \in L_{p}(\mathbb{R})$. Then for the Hilbert transform

$$
H(g, x):=\lim _{\varepsilon \rightarrow 0+} \int_{|x-t| \geq \varepsilon} \frac{g(t)}{x-t} d t, \quad x \in \mathbb{R}
$$


we have

$$
\|H(g)\|_{L_{p}(\mathbb{R})} \leq C\|g\|_{L_{p}(\mathbb{R})}
$$

where $C>0$ is a constant depending upon $p$ only.

Lemma 3.8 (see [7, Theorem 1.4, Theorem 1.6]) Let $w=\exp (-Q) \in \mathcal{F}\left(C^{2}\right), 1 \leq p \leq \infty$ and $\gamma \geq 0$. Then for any $\varepsilon>0$, there exists a polynomial $P$ such that

$$
\left\|(f(x)-P(x))\left(1+x^{2}\right)^{\gamma} w(x)\right\|_{L_{p}(\mathbb{R})}<\varepsilon
$$

Lemma 3.9 Let $w \in \mathcal{F}\left(C^{2}+\right)$ be an Erdös-type weight, that is, $T(x)$ is unbounded. Then for any $M>1$, there exist $x_{M}>0$ and $C_{M}>0$ such that

$$
Q(x) \geq C_{M} x^{M}, \quad x \geq x_{M} .
$$

Proof For every $M>1$, there exists $x_{M}>0$ such that $T(x) \geq M$ for $x \geq x_{M}$, so that $Q^{\prime}(x) / Q(x)=T(x) / x \geq M / x$ for $x \geq x_{M}$. Hence, we see

$$
\log \frac{Q(x)}{Q\left(x_{M}\right)} \geq \log \left(\frac{x}{x_{M}}\right)^{M}, \quad x \geq x_{M}
$$

that is,

$$
Q(x) \geq \frac{Q\left(x_{M}\right)}{\left(x_{M}\right)^{M}} x^{M}, \quad x \geq x_{M} .
$$

Let us put $C_{M}:=Q\left(x_{M}\right) /\left(x_{M}\right)^{M}$.

\section{Proof of Theorem 2.2 by Damelin and Lubinsky methods}

In this section, we assume $w \in \mathcal{F}\left(C^{2}+\right)$. To prove the theorem we need some lemmas, and we will use the Damelin and Lubinsky methods of [3].

Lemma 4.1 (cf. [3, Lemma 3.1]) Let $w \in \mathcal{F}\left(C^{2}+\right)$. Let $0<\alpha<\frac{1}{4}$ and

$$
\sum_{n}(x):=\sum_{\left|x_{k, n}\right| \geq a_{\alpha n}}\left|l_{k, n}(x)\right| w^{-1}\left(x_{k, n}\right) .
$$

Then we have for $|x| \leq a_{\alpha n / 2}$ and $|x| \geq a_{2 n}$,

$$
\sum_{n}(x) w(x) \leq C
$$

Moreover, for $a_{\alpha n / 2} \leq|x| \leq a_{2 n}$,

$$
\sum_{n}(x) w(x) \leq C\left(\log n+a_{n}^{\frac{1}{2}}\left|p_{n}(x) w(x)\right| T^{-\frac{1}{4}}\left(a_{n}\right)\right) .
$$


Proof The proof of [3, Lemma 3.1] holds without the condition (1.2) and the second condition in (1.1) and under the assumption of the quasi-increasingness of $T(x)$. The conditions in Definition 1.1 contain all the conditions in Definition 1.3 except for (1.2) and the second condition in (1.1). We see that in [3, Lemma 3.1] we can replace $T^{*}(x)$ with $T(x)$.

Lemma $4.2([3$, Lemma 3.2]) Let $0<\eta<1$. Let $\psi: \mathbb{R} \mapsto(0, \infty)$ be a continuous function with the following property: For $n \geq 1$, there exist polynomials $R_{n}$ of degree $\leq n$ such that

$$
C_{1} \leq \frac{\psi(t)}{R_{n}(t)} \leq C_{2}, \quad|t| \leq a_{4 n}
$$

Then for $n \geq n_{0}$ and $P \in \mathcal{P}_{n}$,

$$
\sum_{\left|x_{k, n}\right| \leq a_{\eta n}} \lambda_{k, n}\left|P\left(x_{k, n}\right)\right| w^{-1}\left(x_{k, n}\right) \psi\left(x_{k, n}\right) \leq C \int_{-a_{4 n}}^{a_{4 n}}|P(t) w(t)| \psi(t) d t .
$$

Remark 4.3 To prove Lemma 4.7 below, we apply this lemma with $\psi(t)=\phi(t)=(1+$ $\left.t^{2}\right)^{-\beta / 2}, \beta>0$. In fact, when $\phi^{*}(x)=\phi(t), t=a_{4 n} x$, we can approximate $\phi^{*}$ by polynomials $R_{n}^{*} \in \mathcal{P}_{n}$ on $[-1,1]$, that is, for any $\varepsilon>0$ there exists $R_{n}^{*} \in \mathcal{P}_{n}$ such that

$$
\left|\phi^{*}(x)-R_{n}^{*}(x)\right|<\varepsilon, \quad x \in[-1,1] .
$$

Therefore,

$$
\left|\frac{R_{n}^{*}(x)}{\phi^{*}(x)}-1\right|<\frac{\varepsilon}{\phi^{*}(x)}, \quad x \in[-1,1]
$$

and so there exist $C_{1}, C_{2}>0$ such that

$$
C_{1} \leq 1-\frac{\varepsilon}{\phi^{*}(x)} \leq\left|\frac{R_{n}^{*}(x)}{\phi^{*}(x)}\right|<1+\frac{\varepsilon}{\phi^{*}(x)} \leq C_{2}, \quad x \in[-1,1] .
$$

Now, if we set $R_{n}(t)=R_{n}^{*}(x)$, then we have the result.

Lemma 4.4 (cf. [3, Lemma 4.1]) Let $\left\{f_{n}\right\}_{n=1}^{\infty}$ be a sequence of measurable functions from $\mathbb{R} \mapsto \mathbb{R}$ such that for $n \geq 1$,

$$
f_{n}(x)=0, \quad|x|<a_{\frac{n}{9}} ; \quad\left|f_{n}(x)\right| w(x) \leq \phi(x), \quad x \in \mathbb{R} .
$$

Then for $1 \leq p \leq \infty$ and $\Delta>0$, we have

$$
\lim _{n \rightarrow \infty}\left\|L_{n}\left(f_{n}\right) w \Phi^{\Delta+\left(\frac{1}{4}-\frac{1}{p}\right)^{+}}\right\|_{L_{p}(\mathbb{R})}=0 .
$$

Proof Let $|x| \leq a_{\frac{n}{18}}$ or $|x| \geq a_{2 n}$. We use the first inequality of Lemma 4.1 with $\alpha=\frac{1}{9}$, then from the assumption with respect to $f_{n}$, we see that

$$
\left|L_{n}\left(f_{n} ; x\right) w(x)\right| \leq \phi\left(a_{\frac{n}{9}}\right) \sum_{\left|x_{k, n}\right| \geq a \frac{n}{9}}\left|l_{k, n}(x)\right| w^{-1}\left(x_{k, n}\right) w(x) \leq C_{1} \phi\left(a_{\frac{n}{9}}\right) .
$$


So,

$$
\begin{aligned}
\left.\left\|L_{n}\left(f_{n}\right) w \Phi^{\Delta+\left(\frac{1}{4}-\frac{1}{p}\right)^{+}}\right\|_{L_{p}\left(|x| \leq a_{\frac{n}{18}}\right.} \text { or }|x| \geq a_{2 n}\right) & \leq \phi\left(a_{\left.\frac{n}{9}\right)}\left\|\Phi^{\Delta+\left(\frac{1}{4}-\frac{1}{p}\right)^{+}}\right\|_{L_{p}(\mathbb{R})}\right. \\
& \leq C_{2} \phi\left(a_{\frac{n}{9}}\right)=o(1)
\end{aligned}
$$

by Lemma 3.9 (note the definition of $\Phi(x)$ ) and the definition of $\phi$ in (2.4). Next, we let $a_{\frac{n}{18}} \leq|x| \leq a_{2 n}$. From the second inequality in Lemma 4.1, we see that

$$
\left|L_{n}\left(f_{n} ; x\right) w(x)\right| \leq \phi\left(a_{\frac{n}{9}}\right)\left(\log n+a_{n}^{\frac{1}{2}}\left|p_{n}(x)\right| w(x) T^{-\frac{1}{4}}\left(a_{n}\right)\right) .
$$

Also, for this range of $x$, we see that

$$
\Phi(x)=\frac{1}{(1+Q(x))^{\frac{2}{3}} T(x)} \sim \frac{1}{\left(1+Q\left(a_{n}\right)\right)^{\frac{2}{3}} T\left(a_{n}\right)} \sim \frac{T^{\frac{1}{3}}\left(a_{n}\right)}{n^{\frac{2}{3}} T\left(a_{n}\right)}=\delta_{n}
$$

by Lemma 3.2(b). So, for $n$ large enough,

$$
\begin{aligned}
& \left\|L_{n}\left(f_{n}\right) w \Phi^{\Delta+\left(\frac{1}{4}-\frac{1}{p}\right)^{+}}\right\|_{L_{p}\left(a_{\frac{n}{18}} \leq|x| \leq a_{2 n}\right)} \\
& \leq \phi\left(a_{\frac{n}{9}}\right) \log n\left\|\Phi^{\Delta+\left(\frac{1}{4}-\frac{1}{p}\right)^{+}}\right\|_{L_{p}\left(a_{\frac{n}{18}} \leq|x| \leq a_{2 n}\right)} \\
& \quad+\phi\left(a_{\frac{n}{9}}\right) a_{n}^{\frac{1}{2}} T^{-\frac{1}{4}}\left(a_{n}\right)\left\|p_{n}(x) w(x) \Phi^{\Delta+\left(\frac{1}{4}-\frac{1}{p}\right)^{+}}\right\|_{L_{p}\left(a_{\frac{n}{18}} \leq|x| \leq a_{2 n}\right)} .
\end{aligned}
$$

Then since $\Delta>0$, using Lemma 3.1(a), Lemma 2.1(a), and Lemma 3.6, we have

$$
\log n\left\|\Phi^{\Delta+\left(\frac{1}{4}-\frac{1}{p}\right)^{+}}\right\|_{L_{p}\left(\frac{n}{18} \leq|x| \leq a_{2 n}\right)} \leq C \delta_{n}^{\Delta}\left(a_{2 n}-a_{\frac{n}{18}}\right)^{\frac{1}{p}} \log n \leq C
$$

and

$$
\begin{aligned}
& a_{n}^{\frac{1}{2}} T^{-\frac{1}{4}}\left(a_{n}\right)\left\|p_{n} w \Phi^{\Delta+\left(\frac{1}{4}-\frac{1}{p}\right)^{+}}\right\|_{L_{p}\left(a_{\frac{n}{18}} \leq|x| \leq a_{2 n}\right)} \\
& \quad \leq T^{-\frac{1}{4}}\left(a_{n}\right) \delta_{n}^{\Delta} a_{n}^{\frac{1}{p}}\left\{\begin{array}{ll}
1, & 1 \leq p<4 \text { or } p=\infty ; \\
\log (1+n), & 4 \leq p,
\end{array} \quad . C .\right.
\end{aligned}
$$

Therefore, we have by (2.4)

$$
\left\|L_{n}\left(f_{n}\right) w \Phi^{\Delta+\left(\frac{1}{4}-\frac{1}{p}\right)^{+}}\right\|_{L_{p}\left(a_{\frac{n}{18}} \leq|x| \leq a_{2 n}\right)} \leq C_{4} \phi\left(a_{\frac{n}{9}}\right)=o(1) .
$$

Consequently, with (4.2) we have (4.1).

Lemma 4.5 (cf. [3, Lemma 4.2]) Let $1 \leq p \leq \infty$. Let $\left\{g_{n}\right\}_{n=1}^{\infty}$ be a sequence of measurable functions from $\mathbb{R} \mapsto \mathbb{R}$ such that for $n \geq 1$,

$$
g_{n}(x)=0, \quad|x| \geq a_{\frac{n}{9}} ; \quad\left|g_{n}(x)\right| w(x) \leq \phi(x), \quad x \in \mathbb{R} .
$$


Let us suppose

$$
\Delta>\frac{9}{4} \frac{\lambda-1}{3 \lambda-1}
$$

where $\lambda \geq 1$ is defined in Lemma 2.1. Then for $1 \leq p \leq \infty$, we have

$$
\lim _{n \rightarrow \infty}\left\|L_{n}\left(g_{n}\right) w \Phi^{\Delta+\left(\frac{1}{4}-\frac{1}{p}\right)^{+}}\right\|_{L_{p}\left(|x| \geq a_{\frac{n}{8}}\right)}=0 .
$$

Proof Using Lemma 3.5(b) and Lemma 3.4(b), we have for $x \geq a_{\frac{n}{8}}$,

$$
\begin{aligned}
\left|L_{n}\left(g_{n} ; x\right)\right| & \leq \sum_{\left|x_{k, n}\right| \leq a \frac{n}{9}}\left|l_{k, n}(x)\right| w^{-1}\left(x_{k, n}\right) \phi\left(x_{k, n}\right) \\
& \leq C_{1} a_{n}^{\frac{1}{2}}\left|p_{n}(x)\right| \sum_{\left|x_{k, n}\right| \leq a_{\frac{n}{9}}}\left(x_{k, n}-x_{k+1, n}\right) \frac{\left(1-\frac{\left|x_{k, n}\right|}{a_{n}}+\delta_{n}\right)^{\frac{1}{4}}}{\left|x-x_{k, n}\right|} \phi\left(x_{k, n}\right) \\
& \leq C_{2} a_{n}^{\frac{1}{2}}\left|p_{n}(x)\right| \int_{-a_{\frac{n}{9}}}^{a_{\frac{n}{9}}} \frac{\left(1-\frac{|t|}{a_{n}}+\delta_{n}\right)^{\frac{1}{4}}}{|x-t|} \phi(t) d t .
\end{aligned}
$$

Equation (4.6) is shown as follows: First, we see

$$
|x-t| \sim\left|x-x_{k, n}\right|, \quad t \in\left[x_{k+1, n}, x_{k, n}\right] .
$$

Let $|x| \geq a_{\frac{n}{8}}$ and $t \in\left[x_{k+1, n}, x_{k, n}\right]$. Then

$$
\left|\frac{x-t}{x-x_{k, n}}-1\right|=\left|\frac{t-x_{k, n}}{x-x_{k, n}}\right| \leq \frac{x_{k, n}-x_{k+1, n}}{\left|x_{k \pm 2, n}-x_{k, n}\right|} \leq c<1
$$

Now, we use the fact that $x+C \varphi(x), x>0$ is increasing for $0<x \leq a_{n / 2}$, and then

$$
x_{k, n}+C \varphi_{n}\left(x_{k, n}\right) \leq a_{\frac{n}{9}}+C \varphi_{n}\left(a_{\frac{n}{9}}\right) \leq a_{\frac{n}{8}} \leq x .
$$

Here, the second inequality follows from the definition of $\varphi_{n}(x)$ and Lemma 3.1(a), (b). Hence, we have (4.7). Now, we use the monotonicity of $\left(1-\frac{|x|}{a_{n}}+\delta_{n}\right)^{\frac{1}{4}} \phi(x)$. From (4.7) there exists $C>0$ such that for $t \in\left[x_{k+1, n}, x_{k, n}\right]$,

$$
\begin{aligned}
\left(x_{k, n}-x_{k+1, n}\right) \frac{\left(1-\frac{\left|x_{k, n}\right|}{a_{n}}+\delta_{n}\right)^{\frac{1}{4}}}{\left|x-x_{k, n}\right|} \phi\left(x_{k, n}\right) & \leq \int_{x_{k+1, n}}^{x_{k, n}} \frac{\left(1-\frac{|t|}{a_{n}}+\delta_{n}\right)^{\frac{1}{4}}}{\left|x-x_{k, n}\right|} \phi(t) d t \\
& \leq \frac{1}{C} \int_{x_{k+1, n}}^{x_{k, n}} \frac{\left(1-\frac{|t|}{a_{n}}+\delta_{n}\right)^{\frac{1}{4}}}{|x-t|} \phi(t) d t .
\end{aligned}
$$

Hence, (4.6) holds. Next, for $t \in\left[0, a_{\frac{n}{9}}\right]$ and $x \geq a_{\frac{n}{8}}$, we know by Lemma 3.1(a),

$$
1 \leq \frac{a_{n}-t}{x-t} \leq 1+\frac{a_{n}-a_{\frac{n}{8}}}{a_{\frac{n}{8}}-t} \leq 1+\frac{a_{n}-a_{\frac{n}{8}}}{a_{\frac{n}{8}}-a_{\frac{n}{9}}} \leq 1+C \frac{a_{\frac{n}{8}}}{a_{\frac{n}{9}}} \frac{T\left(a_{\frac{n}{9}}\right)}{T\left(a_{\frac{n}{8}}\right)} \leq C_{3}
$$


and

$$
1-\frac{|t|}{a_{n}} \geq C_{4} \frac{1}{T\left(a_{n}\right)} \geq \delta_{n} .
$$

So, we have

$$
\left|L_{n}\left(g_{n} ; x\right)\right| \leq C_{6} a_{n}^{\frac{1}{4}}\left|p_{n}(x)\right| \int_{0}^{a \frac{n}{9}}(x-t)^{-\frac{3}{4}} \phi(t) d t .
$$

Let $t=a_{s}, \frac{n}{9} \geq s \geq 1$. Then, since we know for $x \geq a_{\frac{n}{8}}$,

$$
x-t=x\left(1-\frac{t}{x}\right) \geq a_{\frac{n}{8}}\left(1-\frac{a_{s}}{a_{\frac{9}{8} s}}\right) \geq C_{7} \frac{a_{n}}{T\left(a_{s}\right)},
$$

we obtain

$$
\left|L_{n}\left(g_{n} ; x\right)\right| \leq C_{8} a_{n}^{-\frac{1}{2}}\left|p_{n}(x)\right| \int_{0}^{a_{n}} T^{\frac{3}{4}}(t) \phi(t) d t \leq C_{8} a_{n}^{\frac{1}{2}} T^{\frac{3}{4}}\left(a_{n}\right)\left|p_{n}(x)\right| .
$$

Hence, if $1 \leq \lambda$, then using Lemma 3.6, (3.1) and (2.2), we have

$$
\begin{aligned}
& \left\|L_{n}\left(g_{n}\right) w \Phi^{\Delta+\left(\frac{1}{4}-\frac{1}{p}\right)^{+}}\right\|_{L_{p}\left(|x| \geq a_{\frac{n}{8}}\right)} \\
& \leq C_{9} a_{n}^{\frac{1}{2}} T^{\frac{3}{4}}\left(a_{n}\right) \Phi^{\Delta}\left(a_{\frac{n}{8}}\right)\left\|\Phi^{\left(\frac{1}{4}-\frac{1}{p}\right)^{+}} w p_{n}\right\|_{L_{p}(\mathbb{R})} \\
& \leq C_{10} a_{n}^{\frac{1}{p}} T^{\frac{3}{4}}\left(a_{n}\right)\left(\frac{1}{n T\left(a_{n}\right)}\right)^{\frac{2}{3} \Delta} \begin{cases}1, & 0<p<4 \text { or } p=\infty \\
\log (1+n), & 4 \leq p\end{cases} \\
& \leq C_{11} C(\lambda, \eta) a_{n}^{\frac{1}{p}}\left(\frac{1}{n}\right)^{\frac{2}{3} \frac{3 \lambda+2 \eta-1}{\lambda+1}\left(\Delta-\frac{9}{4} \frac{\lambda+\eta-1}{3 \lambda+2 \eta-1}\right)} \begin{cases}1, & 1 \leq p<4 \text { or } p=\infty ; \\
\log (1+n), & 4 \leq p .\end{cases}
\end{aligned}
$$

Here, we may consider that above estimations hold under the condition (4.4), because that $\eta>0$ can be taken small enough. Then we have (4.5), that is, for $\Delta>\frac{9}{4} \frac{\lambda-1}{3 \lambda-1}$,

$$
\lim _{n \rightarrow \infty}\left\|L_{n}\left(g_{n}\right) w \Phi^{\Delta+\left(\frac{1}{4}-\frac{1}{p}\right)^{+}}\right\|_{L_{p}\left(|x| \geq a_{\frac{n}{8}}\right)}=0 .
$$

Lemma 4.6 (cf. [3, Lemma 4.3]) Let $1<p<\infty$. Let $\sigma: \mathbb{R} \mapsto \mathbb{R}$ be a bounded measurable function. Let $\lambda=\lambda(b) \geq 1$ be defined in Lemma 2.1, and then we suppose

$$
\Delta> \begin{cases}0, & 1<p \leq 2 \\ \frac{3}{2} \frac{(\lambda-1)}{3 \lambda-1} \frac{p-2}{p}, & 2<p \leq 4 \\ \max \left\{\frac{\lambda-1}{3 \lambda-1} \frac{p-1}{p}-\frac{1}{4} \frac{\lambda+1}{3 \lambda-1} \frac{p-4}{p}, 0\right\}, & 4<p .\end{cases}
$$

Then for $1<p<\infty$ and the partial sum $s_{n}$ of the Fourier series, we have

$$
\left\|s_{n}\left[\sigma \phi w^{-1}\right] w \Phi^{\Delta+\left(\frac{1}{4}-\frac{1}{p}\right)^{+}}\right\|_{L_{p}\left(|x| \leq a \frac{n}{8}\right)} \leq C\|\sigma\|_{L_{\infty}(\mathbb{R})}
$$

for $n \geq 1$. Here $C$ is independent of $\sigma$ and $n$. 
Proof We may suppose that $\|\sigma\|_{L_{\infty}(\mathbb{R})}=1$. By (3.3), (3.4) and Lemma 3.5(a),

$$
\left|s_{n}\left[\sigma \phi w^{-1}\right](x)\right| w(x) \leq a_{n}^{\frac{1}{2}}\left(1-\frac{|x|}{a_{n}}\right)^{-\frac{1}{4}} \sum_{j=n-1}^{n}\left|H\left[\sigma \phi p_{j} w\right](x)\right| .
$$

Let us choose $l:=l(n)$ such that $2^{l} \leq \frac{n}{8} \leq 2^{l+1}$. Then we know

$$
2^{l+3} \leq n \leq 2^{l+4} .
$$

Define

$$
\mathcal{I}_{k}=\left[a_{2^{k}}, a_{2^{k+1}}\right], \quad 1 \leq k \leq l+2 .
$$

For $j=n-1, n$ and $x \in \mathcal{I}_{k}$, we split

$$
\begin{aligned}
H\left[\sigma \phi p_{j} w\right](x) w(x) & =\left(\int_{-\infty}^{0}+\int_{0}^{a_{2^{k-1}}}+P . V \cdot \int_{a_{2^{k-1}}}^{a_{2^{k+2}}}+\int_{a_{2^{k+2}}}^{\infty}\right) \frac{\left(\sigma \phi p_{j} w\right)(t)}{x-t} d t \\
: & I_{1}(x)+I_{2}(x)+I_{3}(x)+I_{4}(x) .
\end{aligned}
$$

Here P.V. stands for the principal value. First, we give the estimations of $I_{1}$ and $I_{2}$ for $x \in \mathcal{I}_{k}$. Let $x \in \mathcal{I}_{k}$. Then we have by Lemma 3.5(a) and Lemma 3.6 with $p=1$,

$$
\begin{aligned}
\left|I_{1}(x)\right| & \leq \int_{0}^{\infty} \frac{\left|\left(p_{j} w \phi\right)(-t)\right|}{t+x} \leq C_{1} a_{n}^{-\frac{1}{2}} \int_{0}^{\frac{a_{n}}{2}} \frac{\phi(t)}{t+a_{2}} d t+C_{2} a_{n}^{-1} \int_{\frac{a_{n}}{2}}^{\infty}\left|p_{j}(t)\right| w(t) d t \\
& \leq C_{2}\left(a_{n}^{-\frac{1}{2}}+a_{n}^{-1} a_{n}^{1-\frac{1}{2}}\right) \leq C_{3} a_{n}^{-\frac{1}{2}} .
\end{aligned}
$$

Here we have used

$$
\int_{0}^{\infty} \frac{\phi(t)}{1+t} d t<\infty
$$

By Lemma 3.5(a), and noting $1-x / a_{n} \leq 1-t / a_{n}$ for $x \in \mathcal{I}_{k}$,

$$
\begin{aligned}
\left|I_{2}(x)\right| & \leq \int_{0}^{a_{2} k-1} \frac{\left|\left(p_{j} w \phi\right)(t)\right|}{x-t} d t \leq C_{4} a_{n}^{-\frac{1}{2}} \int_{0}^{a_{2^{k-1}}} \frac{\left(1-\frac{t}{a_{n}}\right)^{-\frac{1}{4}}}{x-t} d t \\
& \leq C_{4} a_{n}^{-\frac{1}{2}}\left(1-\frac{x}{a_{n}}\right)^{-\frac{1}{4}} \int_{0}^{a_{2^{k-1}}} \frac{d t}{x-t} \\
& =C_{4} a_{n}^{-\frac{1}{2}}\left(1-\frac{x}{a_{n}}\right)^{-\frac{1}{4}} \log \left(1-\frac{a_{2^{k-1}}}{x}\right)^{-1} .
\end{aligned}
$$

Using

$$
1-\frac{a_{2^{k-1}}}{x} \geq 1-\frac{a_{2^{k-1}}}{a_{2^{k}}} \geq C \frac{1}{T\left(a_{2^{k}}\right)} \geq C \frac{1}{T(x)},
$$

we can see

$$
\left|I_{2}(x)\right| \leq C_{6} a_{n}^{-\frac{1}{2}}\left(1-\frac{x}{a_{n}}\right)^{-\frac{1}{4}} \log \left(\frac{T(x)}{C}\right) .
$$


Next, we give an estimation of $I_{4}$ for $x \in \mathcal{I}_{k}$. Let $x \in \mathcal{I}_{k}$. From Lemma 3.5(a) again,

$$
\begin{aligned}
\left|I_{4}(x)\right| \leq & \int_{a_{2^{k+2}}}^{2 a_{2^{k+2}}} \frac{\left|\left(p_{j} w \phi\right)(t)\right|}{t-x} d t+C \int_{2 a_{2^{k+2}}}^{\infty} \frac{\left|\left(p_{j} w \phi\right)(t)\right|}{t} d t \quad(\text { by } t \leq 2(t-x)) \\
\leq & C_{7}\left(a_{n}^{-\frac{1}{2}} \int_{a_{2^{k+2}}}^{2 a_{2^{k+2}}}\left|1-\frac{t}{a_{n}}\right|^{-\frac{1}{4}} \frac{d t}{t-x}\right. \\
& \left.+a_{n}^{-\frac{1}{2}} \int_{2 a_{2^{k+2}}}^{\max \left\{2 a_{2^{k+2}}, \frac{1}{2} a_{n}\right\}} \frac{\phi(t)}{t} d t+\int_{\frac{1}{2} a_{n}}^{\infty} \frac{\left|\left(p_{j} w\right)(t)\right|}{t} d t\right) \\
\leq & C_{7}\left(a_{n}^{-\frac{1}{2}} \int_{a_{2^{k+2}}}^{2 a_{2^{k+2}}}\left|1-\frac{t}{a_{n}}\right|^{-\frac{1}{4}} \frac{d t}{t-x}+C a_{n}^{-\frac{1}{2}}+a_{n}^{-1} a_{n}^{1-\frac{1}{2}}\right)
\end{aligned}
$$

(by (4.14) and Lemma 3.6 with $p=1$ )

$$
\leq C_{8} a_{n}^{-\frac{1}{2}}[J+1]
$$

where

$$
J:=\int_{a_{2^{k+2}}}^{2 a_{2^{k+2}}}\left|1-\frac{t}{a_{n}}\right|^{-\frac{1}{4}} \frac{d t}{t-x} .
$$

Since, if

$$
\left|1-\frac{t}{a_{n}}\right| \leq \frac{1}{2}\left(1-\frac{x}{a_{n}}\right)
$$

then we see

$$
|t-x|=a_{n}\left|\left(1-\frac{x}{a_{n}}\right)-\left(1-\frac{t}{a_{n}}\right)\right| \geq \frac{a_{n}}{2}\left(1-\frac{x}{a_{n}}\right) .
$$

Now, we have

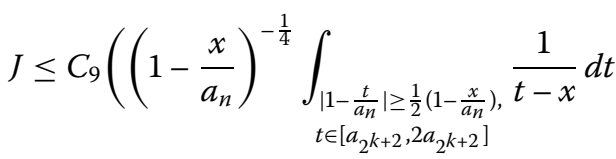

$$
\begin{aligned}
& \left.+a_{n}^{-1}\left(1-\frac{x}{a_{n}}\right)^{-1} \int_{\substack{\left|1-\frac{t}{a_{n}}\right| \leq \frac{1}{2}\left(1-\frac{x}{a_{n}}\right), t \in\left[a_{2} k+2,2 a_{2} k+2\right]}}\left|1-\frac{t}{a_{n}}\right|^{-\frac{1}{4}} d t\right) \\
& \leq C_{10}\left(\left(1-\frac{x}{a_{n}}\right)^{-\frac{1}{4}} \log \left(1+\frac{a_{2^{k+2}}}{a_{2^{k+2}}-a_{2^{k+1}}}\right)\right. \\
& \left.+\left(1-\frac{x}{a_{n}}\right)^{-1} \int_{|1-s| \leq \frac{1}{2}\left(1-\frac{x}{a_{n}}\right)}|1-s|^{-\frac{1}{4}} d s\right) \\
& \leq C_{10}\left(\left(1-\frac{x}{a_{n}}\right)^{-\frac{1}{4}} \log \left(1+C T\left(a_{2^{k+2}}\right)\right)+\frac{4}{3}\left(\frac{1}{2}\left(1-\frac{x}{a_{n}}\right)\right)^{\frac{3}{4}}\left(1-\frac{x}{a_{n}}\right)^{-1}\right) \\
& \leq C_{11}\left(1-\frac{x}{a_{n}}\right)^{-\frac{1}{4}} \log (C T(x)) \text {. }
\end{aligned}
$$


So, from (4.16) we have

$$
\left|I_{4}(x)\right| \leq C_{12} a_{n}^{-\frac{1}{2}}\left(1-\frac{x}{a_{n}}\right)^{-\frac{1}{4}} \log (C T(x))
$$

Therefore, from (4.13), (4.15) and (4.17), we have

$$
\left|I_{1}+I_{2}+I_{4}\right| \leq C_{13} a_{n}^{-\frac{1}{2}}\left(1-\frac{x}{a_{n}}\right)^{-\frac{1}{4}} \log (C T(x))
$$

Hence, with (4.10), (4.12) we have

$$
\begin{aligned}
& \left\|s_{n}\left[\sigma \phi w^{-1}\right] w \Phi^{\Delta+\left(\frac{1}{4}-\frac{1}{p}\right)^{+}}\right\|_{L_{p}\left(\mathcal{I}_{k}\right)} \\
& \leq C_{14} \Phi^{\Delta+\left(\frac{1}{4}-\frac{1}{p}\right)^{+}}\left(a_{2^{k}}\right)\left(\left(1-\frac{a_{2^{k+1}}}{a_{n}}\right)^{-\frac{1}{2}} \log \left(C T\left(a_{2^{k+1}}\right)\right)\left(a_{2^{k+1}}-a_{2^{k}}\right)^{\frac{1}{p}}\right. \\
& \left.\quad+a_{n}^{\frac{1}{2}}\left(1-\frac{a_{2^{k+1}}}{a_{n}}\right)^{-\frac{1}{4}} \sum_{j=n-1}^{n}\left\|P \cdot V \cdot \int_{a_{2^{k-1}}}^{a_{2^{k+2}}} \frac{\left(\sigma \phi p_{j} w\right)(t)}{x-t} d t\right\|_{L_{p}\left(\mathcal{I}_{k}\right)}\right) .
\end{aligned}
$$

We must estimate the $L_{p}$-norm with respect to $I_{3}$, that is, $\left\|P . V \cdot \int_{a^{k-1}}^{a_{2^{k+2}}} \frac{\left(\sigma \phi p_{j} w\right)(t)}{x-t} d t\right\|_{L_{p}\left(\mathcal{I}_{k}\right)}$. We use M. Riesz's theorem on the boundedness of the Hilbert transform from $L_{p}(\mathbb{R})$ to $L_{p}(\mathbb{R})$ (Lemma 3.7) to deduce that by Lemma 3.5(a) and the boundedness of $|\sigma \phi|$,

$$
\begin{aligned}
\left\|P . V . \int_{a_{2^{k-1}}}^{a_{2^{k+2}}} \frac{\left(\sigma \phi p_{j} w\right)(t)}{x-t} d t\right\|_{L_{p}\left(\mathcal{I}_{k}\right)} & \leq C_{15}\left(\int_{a_{2^{k-1}}}^{a_{2^{k+2}}}\left|\left(\sigma \phi p_{j} w\right)(t)\right|^{p} d t\right)^{\frac{1}{p}} \\
& \leq C_{16} a_{n}^{-\frac{1}{2}}\left(1-\frac{a_{2^{k+2}}}{a_{n}}\right)^{-\frac{1}{4}}\left(a_{2^{k+2}}-a_{2^{k-1}}\right)^{\frac{1}{p}} .
\end{aligned}
$$

So, by (4.18) and (4.19) we conclude

$$
\begin{aligned}
& \left\|s_{n}\left[\sigma \phi w^{-1}\right] w \Phi^{\Delta+\left(\frac{1}{4}-\frac{1}{p}\right)^{+}}\right\|_{L_{p}\left(\mathcal{I}_{k}\right)} \\
& \quad \leq C_{18} \Phi^{\Delta+\left(\frac{1}{4}-\frac{1}{p}\right)^{+}}\left(a_{2^{k}}\right)\left(1-\frac{a_{2^{k+1}}}{a_{n}}\right)^{-\frac{1}{2}} \log \left(C T\left(a_{2^{k+1}}\right)\right)\left(a_{2^{k+1}}-a_{2^{k}}\right)^{\frac{1}{p}} .
\end{aligned}
$$

Noting (4.11), we see $n \geq 2^{l+3}$ for $k \leq l$, so

$$
1-\frac{a_{2^{k+1}}}{a_{n}} \geq 1-\frac{a_{2^{k+1}}}{a_{2^{k+3}}} \geq C_{19} \frac{1}{T\left(a_{2^{k}}\right)} \quad \text { and } \quad a_{2^{k+1}}-a_{2^{k}} \leq C_{20} \frac{a_{2^{k}}}{T\left(a_{2^{k}}\right)} .
$$

On the other hand, using Lemma 3.2(b), we see $\Phi\left(a_{t}\right) \sim \delta_{t}$. Hence, we have

$$
\begin{aligned}
\Phi^{\Delta+\left(\frac{1}{4}-\frac{1}{p}\right)^{+}}\left(a_{2^{k}}\right) & \sim \delta_{2^{k}}^{\Delta+\left(\frac{1}{4}-\frac{1}{p}\right)^{+}}=\left(\frac{1}{2^{k} T\left(a_{2^{k}}\right)}\right)^{\frac{2}{3}\left(\Delta+\left(\frac{1}{4}-\frac{1}{p}\right)^{+}\right)} \\
& = \begin{cases}\left(\frac{1}{2^{k} T\left(a_{2^{k}}\right)}\right)^{\frac{2}{3} \Delta}, & 0<p \leq 4 ; \\
\left(\frac{1}{2^{k} T\left(a_{2^{k}}\right)}\right)^{\frac{2}{3}\left(\Delta+\left(\frac{1}{4}-\frac{1}{p}\right)\right)}, & 4<p .\end{cases}
\end{aligned}
$$


Hence, from (4.20) we have

$$
\begin{aligned}
& \left\|s_{n}\left[\sigma \phi w^{-1}\right] w \Phi^{\Delta+\left(\frac{1}{4}-\frac{1}{p}\right)^{+}}\right\|_{L_{p}\left(\mathcal{I}_{k}\right)} \\
& \quad \leq C_{19} \Phi^{\Delta+\left(\frac{1}{4}-\frac{1}{p}\right)^{+}}\left(a_{2^{k}}\right) T^{\frac{1}{2}}\left(a_{2^{k}}\right) \log \left(C T\left(a_{2^{k+1}}\right)\right)\left(\frac{a_{2^{k}}}{T\left(a_{2^{k}}\right)}\right)^{\frac{1}{p}} \\
& \quad \leq C_{19} \log \left(C T\left(a_{2^{k+1}}\right)\right) a_{2^{k}}^{\frac{1}{p}} \begin{cases}\left(\frac{1}{2^{k}}\right)^{\frac{2}{3} \Delta} T^{-\frac{2}{3} \Delta+\frac{1}{2}-\frac{1}{p}}\left(a_{2^{k}}\right), & 1<p \leq 4 ; \\
\left(\frac{1}{2^{k}}\right)^{\frac{2}{3}\left(\Delta+\left(\frac{1}{4}-\frac{1}{p}\right)\right)} T^{-\frac{2}{3} \Delta+\frac{1}{3}\left(1-\frac{1}{p}\right)}\left(a_{2^{k}}\right), & 4<p .\end{cases}
\end{aligned}
$$

From Lemma 2.1 (2.2), we know

$$
T^{-\frac{2}{3} \Delta+\frac{1}{2}-\frac{1}{p}}\left(a_{2^{k}}\right) \leq C_{1} C(\lambda, \eta)\left(2^{k}\right)^{\frac{2(\eta+\lambda-1)}{\lambda+1} \max \left\{-\frac{2}{3} \Delta+\frac{1}{2}-\frac{1}{p}, 0\right\}},
$$

and

$$
T^{-\frac{2}{3} \Delta+\frac{1}{3}\left(1-\frac{1}{p}\right)}\left(a_{2^{k}}\right) \leq C_{2} C(\lambda, \eta)\left(2^{k}\right)^{\frac{2(\eta+\lambda-1)}{\lambda+1} \max \left\{-\frac{2}{3} \Delta+\frac{1}{3}\left(1-\frac{1}{p}\right), 0\right\}} .
$$

Therefore, we continue with Lemma 2.1(a) as

$$
\begin{aligned}
\leq & C_{20} C(\lambda, \eta) \log \left(C T\left(a_{2^{k+1}}\right)\right) \\
& \times \begin{cases}\left(\frac{1}{2^{k}}\right)^{\frac{2}{3} \Delta-\frac{\eta}{p}-\frac{2(\eta+\lambda-1)}{\lambda+1} \max \left\{-\frac{2}{3} \Delta+\frac{1}{2}-\frac{1}{p}, 0\right\}}, & 1<p \leq 4 ; \\
\left(\frac{1}{2^{k}}\right)^{\frac{2}{3}\left(\Delta+\left(\frac{1}{4}-\frac{1}{p}\right)\right)-\frac{\eta}{p}-\frac{2(\eta+\lambda-1)}{\lambda+1} \max \left\{-\frac{2}{3} \Delta+\frac{1}{3}\left(1-\frac{1}{p}\right), 0\right\}}, & 4<p .\end{cases}
\end{aligned}
$$

First, let $1<p \leq 4$. Then (4.8), that is,

$$
\Delta> \begin{cases}0, & 1<p \leq 2 \\ \frac{3}{2} \frac{\lambda-1}{3 \lambda-1} \frac{p-2}{p}, & 2<p \leq 4\end{cases}
$$

implies

$$
\Delta>\frac{3}{2} \frac{\lambda-1}{3 \lambda-1} \frac{p-2}{p} \text { and } \Delta>0
$$

iff

$$
\frac{2}{3} \Delta-\frac{2(\lambda-1)}{\lambda+1}\left(-\frac{2}{3} \Delta+\frac{1}{2}-\frac{1}{p}\right)>0 \text { and } \quad \Delta>0
$$

iff

$$
\frac{2}{3} \Delta-\frac{2(\lambda-1)}{\lambda+1} \max \left\{-\frac{2}{3} \Delta+\frac{1}{2}-\frac{1}{p}, 0\right\}>0
$$

This means that there exists a positive constant $\eta_{1}>0$ small enough such that

$$
A\left(\eta_{1}\right):=\frac{2}{3} \Delta-\frac{\eta_{1}}{p}-\frac{2\left(\eta_{1}+\lambda-1\right)}{\lambda+1} \max \left\{-\frac{2}{3} \Delta+\frac{1}{2}-\frac{1}{p}, 0\right\}>0 .
$$


Now, let $p>4$. Then (4.8), that is,

$$
\Delta>\frac{\lambda-1}{3 \lambda-1} \frac{p-1}{p}-\frac{1}{4} \frac{\lambda+1}{3 \lambda-1} \frac{p-4}{p}
$$

implies

$$
\Delta>\frac{\lambda-1}{3 \lambda-1}\left(1-\frac{1}{p}\right)-\frac{\lambda+1}{3 \lambda-1}\left(\frac{1}{4}-\frac{1}{p}\right) \text { and } \Delta+\frac{1}{4}-\frac{1}{p}>0
$$

iff

$$
\frac{2}{3}\left(\Delta+\left(\frac{1}{4}-\frac{1}{p}\right)\right)-\frac{2(\lambda-1)}{\lambda+1}\left(-\frac{2}{3} \Delta+\frac{1}{3}\left(1-\frac{1}{p}\right)\right)>0
$$

and

$$
\frac{2}{3}\left(\Delta+\left(\frac{1}{4}-\frac{1}{p}\right)\right)>0
$$

iff

$$
\frac{2}{3}\left(\Delta+\left(\frac{1}{4}-\frac{1}{p}\right)\right)-\frac{2(\lambda-1)}{\lambda+1} \max \left\{-\frac{2}{3} \Delta+\frac{1}{3}\left(1-\frac{1}{p}\right), 0\right\}>0 .
$$

Similarly to the previous case, this means that there exists a positive constant $\eta_{2}>0$ small enough such that

$$
B\left(\eta_{2}\right):=\frac{2}{3}\left(\Delta+\left(\frac{1}{4}-\frac{1}{p}\right)\right)-\frac{\eta_{2}}{p}-\frac{2\left(\eta_{2}+\lambda-1\right)}{\lambda+1} \max \left\{-\frac{2}{3} \Delta+\frac{1}{3}\left(1-\frac{1}{p}\right), 0\right\}>0 .
$$

Now, we estimate $I_{p, k}$. From (4.21), we have

$$
\begin{aligned}
& \left\|s_{n}\left[\sigma \phi w^{-1}\right] w \Phi^{\Delta+\left(\frac{1}{4}-\frac{1}{p}\right)^{+}}\right\|_{L_{p}\left(\mathcal{I}_{k}\right)} \\
& \quad \leq C_{20} C(\lambda, \eta) \log \left(C T\left(a_{2^{k+1}}\right)\right) \begin{cases}\left(\frac{1}{2^{k}}\right)^{A(\eta)}, & 1<p \leq 4 ; \\
\left(\frac{1}{2^{k}}\right)^{B(\eta)}, & 4<p .\end{cases}
\end{aligned}
$$

For $\eta>0$ small enough, we can see $A(\eta)>A\left(\eta_{1}\right)>0$ and $B(\eta)>B\left(\eta_{2}\right)>0$. Let $\tau:=$ $\min \left\{A\left(\eta_{1}\right), B\left(\eta_{2}\right)\right\} / 2$. Then for small enough $\eta>0$, we have

$$
\begin{aligned}
\left\|s_{n}\left[\sigma \phi w^{-1}\right] w \Phi^{\Delta+\left(\frac{1}{4}-\frac{1}{p}\right)^{+}}\right\|_{L_{p}\left(\mathcal{I}_{k}\right)} & \leq C_{20} C(\lambda, \eta) \log \left(C T\left(a_{2^{k+1}}\right)\right)\left(\frac{1}{2^{k}}\right)^{2 \tau} \\
& \leq C_{21} C(\lambda, \eta)\left(\frac{1}{2^{k}}\right)^{\tau},
\end{aligned}
$$

because we see that for all $k>0$,

$$
\log \left(C T\left(a_{2^{k+1}}\right)\right)\left(\frac{1}{2^{k}}\right)^{\tau}<C_{22}
$$


Therefore, under the conditions (4.8) we have

$$
\begin{aligned}
\left\|s_{n}\left[\sigma \phi w^{-1}\right] w \Phi^{\Delta+\left(\frac{1}{4}-\frac{1}{p}\right)^{+}}\right\|_{L_{p}\left(a_{2} \leq|x| \leq a_{n}\right)}^{p} & \leq \sum_{k=1}^{l}\left\|s_{n}\left[\sigma \phi w^{-1}\right] w \Phi^{\Delta+\left(\frac{1}{4}-\frac{1}{p}\right)^{+}}\right\|_{L_{p}\left(\mathcal{I}_{k}\right)}^{p} \\
& \leq C_{21} C(\lambda, \eta) \sum_{k=1}^{l}\left(\frac{1}{2^{k}}\right)^{\tau} \leq C_{23} C(\lambda, \eta) .
\end{aligned}
$$

The estimation of

$$
\left\|s_{n}\left[\sigma \phi w^{-1}\right] w \Phi^{\Delta+\left(\frac{1}{4}-\frac{1}{p}\right)^{+}}\right\|_{L_{p}\left(|x| \leq a_{2}\right)}^{p}
$$

is similar. In fact, for $x \in\left[-a_{2}, a_{2}\right]$, we split

$$
H\left[\sigma \phi p_{j} w\right](x)=\left(\int_{-\infty}^{-2 a_{2}}+P . V . \int_{-2 a_{2}}^{2 a_{2}}+\int_{2 a_{2}}^{\infty}\right) \frac{\left(\sigma \phi p_{j} w\right)(t)}{x-t} d t
$$

Here we see that

$$
\begin{aligned}
\left|\int_{-\infty}^{-2 a_{2}} \frac{\left(\sigma \phi p_{j} w\right)(t)}{x-t} d t\right| & =\left|\int_{2 a_{2}}^{\infty} \frac{\left(\sigma \phi p_{j} w\right)(-t)}{x+t} d t\right| \leq\left|\int_{2 a_{2}}^{\infty} \frac{\left(\sigma \phi p_{j} w\right)(-t)}{t-a_{2}} d t\right| \\
& =\left|\int_{0}^{\infty} \frac{\left(\sigma \phi p_{j} w\right)\left(-s-2 a_{2}\right)}{s+a_{2}} d t\right|
\end{aligned}
$$

and

$$
\begin{aligned}
\left|\int_{2 a_{2}}^{\infty} \frac{\left(\sigma \phi p_{j} w\right)(t)}{x-t} d t\right| & =\left|\int_{2 a_{2}}^{\infty} \frac{\left(\sigma \phi p_{j} w\right)(t)}{t-x} d t\right| \leq\left|\int_{2 a_{2}}^{\infty} \frac{\left(\sigma \phi p_{j} w\right)(t)}{t-a_{2}} d t\right| \\
& =\left|\int_{0}^{\infty} \frac{\left(\sigma \phi p_{j} w\right)\left(s+2 a_{2}\right)}{s+a_{2}} d s\right|
\end{aligned}
$$

So, we can estimate $\int_{-\infty}^{-2 a_{2}}$ and $\int_{2 a_{2}}^{\infty}$ as we did $I_{1}$ before (see (4.12)). We can estimate the second integral as follows: By M. Riesz's theorem,

$$
\left\|P . V . \int_{-2 a_{2}}^{2 a_{2}} \frac{\left(\sigma \phi p_{j} w\right)(t)}{x-t} d t\right\|_{L_{p}\left(|t| \leq 2 a_{2}\right)}^{p} \leq C \int_{-2 a_{2}}^{2 a_{2}}\left|\left(\sigma \phi p_{j} w\right)(t)\right|^{p} d t \leq C a_{n}^{-\frac{p}{2}} \leq C .
$$

Now, under the assumption (4.8), we can select $\eta_{0}>0$ small enough such that

$$
\Delta> \begin{cases}0, & 1<p \leq 2 ; \\ \frac{3}{2} \frac{\lambda+\eta_{0}-1}{3 \lambda+2 \eta_{0}-1} \frac{p-2}{p}, & 2<p \leq 4 \\ \max \left\{\frac{\lambda+\eta_{0}-1}{3 \lambda+2 \eta_{0}-1} \frac{p-1}{p}-\frac{1}{4} \frac{\lambda+1}{3 \lambda+2 \eta_{0}-1} \frac{p-4}{p}, 0\right\}, & 4<p .\end{cases}
$$

Consequently, from (4.22) with $\eta_{0}$ we have the result (4.9).

Let $0<\alpha<1$, then for $g_{n}$ in Lemma 4.5 we estimate $L_{n}\left(g_{n}\right)$ over $\left[-a_{\alpha n}, a_{\alpha n}\right]$. 
Lemma 4.7 (cf. [3, Lemma 4.4]) Let $1<p<\infty$ and $0<\varepsilon<1$. Let $\left\{g_{n}\right\}$ be as in Lemma 4.4, but we exchange (4.3) with

$$
\left|g_{n}(x) w(x)\right| \leq \varepsilon \phi(x), \quad x \in \mathbb{R}, n \geq 1 .
$$

Then for $1<p<\infty$,

$$
\lim \sup _{n \rightarrow \infty}\left\|L_{n}\left(g_{n}\right) w \Phi^{\Delta+\left(\frac{1}{4}-\frac{1}{p}\right)^{+}}\right\|_{L_{p}\left(|x| \leq a_{n}\right)} \leq C \varepsilon
$$

Proof Let

$$
\chi_{n}:=\chi_{\left[-a_{\frac{n}{8}, a_{n}}\right]} ; \quad h_{n}:=\operatorname{sign}\left(L_{n}\left(g_{n}\right)\right)\left|L_{n}\left(g_{n}\right)\right|^{p-1} \chi_{n} w^{p-2} \Phi^{\left(\Delta+\left(\frac{1}{4}-\frac{1}{p}\right)^{+}\right) p}
$$

and

$$
\sigma_{n}:=\operatorname{sign} s_{n}\left[h_{n}\right]
$$

We shall show that

$$
\left\|L_{n}\left(g_{n}\right) w \Phi^{\Delta+\left(\frac{1}{4}-\frac{1}{p}\right)^{+}}\right\|_{L_{p}\left(|x| \leq a_{\frac{n}{8}}\right)} \leq \varepsilon\left\|s_{n}\left[\sigma_{n} \phi w^{-1}\right] w \Phi^{\Delta+\left(\frac{1}{4}-\frac{1}{p}\right)^{+}}\right\|_{L_{p}\left(|x| \leq a_{n} \frac{n}{8}\right.} .
$$

Then from Lemma 4.5 we will conclude (4.22). Using orthogonality of $f-s_{n}[f]$ to $\mathcal{P}_{n-1}$, and the Gauss quadrature formula, we see that

$$
\begin{aligned}
& \left\|L_{n}\left(g_{n}\right) w \Phi^{\Delta+\left(\frac{1}{4}-\frac{1}{p}\right)^{+}}\right\|_{L_{p}\left(|x| \leq a_{\frac{n}{8}}\right)}^{p} \\
& \quad=\int_{\mathbb{R}} L_{n}\left(g_{n}\right)(x) h_{n}(x) w^{2}(x) d x \\
& =\int_{\mathbb{R}} L_{n}\left(g_{n}\right)(x) s_{n}\left[h_{n}\right](x) w^{2}(x) d x=\sum_{j=1}^{n} \lambda_{j, n} g_{n}\left(x_{j, n}\right) s_{n}\left[h_{n}\right]\left(x_{j, n}\right) \\
& =\sum_{\left|x_{j, n}\right| \leq a_{\frac{n}{8}}} \lambda_{j, n} g_{n}\left(x_{j, n}\right) s_{n}\left[h_{n}\right]\left(x_{j, n}\right) \quad\left(\text { see }(4.4), \text { that is, the definition of } g_{n}\right) \\
& \quad \leq \varepsilon \sum_{\left|x_{j, n}\right| \leq a_{\frac{n}{8}}} \lambda_{j, n} w^{-1}\left(x_{j, n}\right) \phi\left(x_{j, n}\right)\left|s_{n}\left[h_{n}\right]\left(x_{j, n}\right)\right| .
\end{aligned}
$$

Here, if we use Lemma 4.2 with $\psi=\phi$, we continue as

$$
\begin{aligned}
& \leq C \varepsilon \int_{\mathbb{R}}\left|s_{n}\left[h_{n}\right](x)\right| \phi(x) w(x) d x \\
& =C \varepsilon \int_{\mathbb{R}} s_{n}\left[h_{n}\right](x) \sigma_{n} \phi(x) w^{-1}(x) w^{2}(x) d x=C \varepsilon \int_{\mathbb{R}} h_{n}(x) s_{n}\left[\sigma_{n} \phi w^{-1}\right](x) w^{2}(x) d x \\
& =C \varepsilon \int_{-\frac{\pi}{8}}^{a_{\frac{n}{8}}} h_{n}(x) s_{n}\left[\sigma_{n} \phi w^{-1}\right](x) w^{2}(x) d x .
\end{aligned}
$$


Using Hölder's inequality with $q=p /(p-1)$, we continue this as

$$
\begin{aligned}
& \leq C \varepsilon\left(\int_{-a_{\frac{n}{8}}}^{a_{\frac{n}{8}}}\left|h_{n}(x) w(x) \Phi^{-\left(\Delta+\left(\frac{1}{4}-\frac{1}{p}\right)^{+}\right)}(x)\right|^{q} d x\right)^{1 / q}\left(\int_{-a_{\frac{n}{8}}}^{a_{\frac{n}{8}}}\left|s_{n}\left[\sigma_{n} \phi w^{-1}\right] w \Phi^{\Delta+\left(\frac{1}{4}-\frac{1}{p}\right)^{+}}\right|^{p} d x\right)^{\frac{1}{p}} \\
& =C \varepsilon\left\|L_{n}\left(g_{n}\right) w \Phi^{\Delta+\left(\frac{1}{4}-\frac{1}{p}\right)^{+}}\right\|_{L_{p}\left(|x| \leq a_{\frac{n}{8}}\right)}^{p-1}\left\|s_{n}\left[\sigma_{n} \phi w^{-1} w \Phi^{\Delta+\left(\frac{1}{4}-\frac{1}{p}\right)^{+}}\right]\right\|_{L_{p}\left(|x| \leq a_{\frac{n}{8}}\right)^{\prime}} .
\end{aligned}
$$

Cancellation of $\left\|L_{n}\left(g_{n}\right) w \Phi^{\Delta+\left(\frac{1}{4}-\frac{1}{p}\right)^{+}}\right\|_{L_{p}\left(|x| \leq a_{\frac{n}{8}}\right)}^{p-1}$ gives (4.23).

Proof of Theorem 2.2 In proving the theorem, we split our functions into pieces that vanish inside or outside $\left[-a_{\frac{n}{9}}, a_{\frac{n}{9}}\right]$. Throughout, we let $\chi_{S}$ denote the characteristic function of a set $S$. Also, we set for some fixed $\beta>0$,

$$
\phi(x)=\left(1+x^{2}\right)^{-\beta / 2},
$$

and suppose (2.5). We note that (2.5) means (4.8). Let $0<\varepsilon<1$. We can choose a polynomial $P$ such that

$$
\left\|(f-P) w \phi^{-1}\right\|_{L_{\infty}(\mathbb{R})} \leq \varepsilon
$$

(see Lemma 3.8). Then we have

$$
\begin{aligned}
& \left\|\left(f-L_{n}(f)\right) w \Phi^{\Delta+\left(\frac{1}{4}-\frac{1}{p}\right)^{+}}\right\|_{L_{p}(\mathbb{R})} \\
& \quad \leq\left\|(f-P) w \Phi^{\Delta+\left(\frac{1}{4}-\frac{1}{p}\right)^{+}}\right\|_{L_{p}(\mathbb{R})}+\left\|L_{n}(P-f) w \Phi^{\Delta+\left(\frac{1}{4}-\frac{1}{p}\right)^{+}}\right\|_{L_{p}(\mathbb{R})} \\
& \quad \leq \varepsilon\left\|\phi \Phi^{\Delta+\left(\frac{1}{4}-\frac{1}{p}\right)^{+}}\right\|_{L_{p}(\mathbb{R})}+\left\|L_{n}(P-f) w \Phi^{\Delta+\left(\frac{1}{4}-\frac{1}{p}\right)^{+}}\right\|_{L_{p}(\mathbb{R})} \\
& \quad \leq C \varepsilon+\left\|L_{n}(P-f) w \Phi^{\Delta+\left(\frac{1}{4}-\frac{1}{p}\right)^{+}}\right\|_{L_{p}(\mathbb{R})^{*}}
\end{aligned}
$$

Here we used that

$$
\left\|\phi \Phi^{\Delta+\left(\frac{1}{4}-\frac{1}{p}\right)^{+}}\right\|_{L_{p}(\mathbb{R})}<\infty
$$

because $\Delta>0$ and $\Phi^{-1}$ grows faster than any power of $x$ (see Lemma 3.9). Next, let

$$
\chi_{n}:=\chi\left[-a_{\frac{n}{9}}, a_{\frac{n}{9}}\right]
$$

and write

$$
P-f=(P-f) \chi_{n}+(P-f)\left(1-\chi_{n}\right)=: g_{n}+f_{n} .
$$

By Lemma 4.4 we have

$$
\lim _{n \rightarrow \infty}\left\|L_{n}\left(f_{n}\right) w \Phi^{\Delta+\left(\frac{1}{4}-\frac{1}{4}\right)^{+}}\right\|_{L_{p}(\mathbb{R})}=0 .
$$

By Lemma 4.5 we have

$$
\lim _{n \rightarrow \infty}\left\|L_{n}\left(g_{n}\right) w \Phi^{\Delta+\left(\frac{1}{4}-\frac{1}{4}\right)^{+}}\right\|_{L_{p}\left(|x| \geq a_{\frac{n}{8}}\right)}=0,
$$


and by Lemma 4.7 ,

$$
\lim \sup _{n \rightarrow \infty}\left\|L_{n}\left(g_{n}\right) w \Phi^{\Delta+\left(\frac{1}{4}-\frac{1}{p}\right)^{+}}\right\|_{L_{p}\left(|x| \leq a \frac{n}{8}\right)} \leq C \varepsilon
$$

Here we take $\varepsilon>0$ as $\varepsilon \rightarrow 0$, then with (4.24) we have the result.

\section{Proof of Theorem 2.4}

Lemma 5.1 ( $c f$. [3, Lemma 3.1]) Let $w \in \mathcal{F}\left(C^{2}+\right)$. Let $0<\alpha<\frac{1}{4}$ and

$$
\sum_{n}(x):=\sum_{\left|x_{k, n}\right| \geq a_{\alpha n}}\left|l_{k, n}(x)\right| w^{-1}\left(x_{k, n}\right) .
$$

Then we have for $x \in \mathbb{R}$,

$$
\sum_{n}(x) w(x) \Phi^{1 / 4}(x) \leq C \log n
$$

Proof From Lemma 4.1 and Lemma 3.6 with $p=\infty$, we have the result easily.

Lemma 5.2 Let $w \in \mathcal{F}\left(C^{2}+\right)$. Let $0<\alpha<\frac{1}{4}$ and

$$
\sum_{n}^{\prime}(x):=\sum_{\left|x_{k, n}\right| \leq a_{\alpha n}}\left|l_{k, n}(x)\right| w^{-1}\left(x_{k, n}\right) .
$$

Then we have

$$
\sum_{n}^{\prime}(x) w(x) \Phi(x)^{3 / 4} \leq C \log n
$$

Proof By Lemma 3.5(c), Lemma 3.4(d) and Lemma 3.5(b),

$$
\begin{aligned}
\sum_{n}^{\prime}(x) & =\sum_{\left|x_{k, n}\right| \leq a_{\alpha n}}\left|l_{k, n}(x)\right| w^{-1}\left(x_{k, n}\right) \\
& =\frac{\left|p_{n}(x)\right|}{\left|x-x_{j x, n}\right|\left|P_{n}^{\prime}\left(x_{j, n}\right)\right| w\left(x_{j, n}\right)}+\sum_{\substack{\left|x_{k, n}\right| \leq a_{\alpha n}, k \neq j j_{x}}} \frac{\left|p_{n}(x)\right|}{\left|x-x_{k, n}\right|\left|P_{n}^{\prime}\left(x_{k, n}\right)\right| w\left(x_{k, n}\right)} \\
& \leq C w(x)^{-1}+a_{n}^{1 / 2}\left|p_{n}(x)\right| \sum_{\substack{\left|x_{k, n}\right| \leq a_{\alpha n}, k \neq j j_{x}}} \frac{\varphi_{n}\left(x_{k, n}\right)\left(1-\frac{\left|x_{k, n}\right|}{a_{n}}\right)}{\left|x-x_{k, n}\right|} \\
& \sim C w(x)^{-1}+\frac{a_{n}^{3 / 2}}{n}\left|p_{n}(x)\right| \sum_{\substack{\left|x_{k, n}\right| \leq a_{\alpha n}, k \neq j_{x}}} \frac{1-\frac{\left|x_{k, n}\right|}{a_{2 n}}}{\sqrt{1-\frac{x_{k, n} \mid}{a_{n}}}}\left(1-\frac{\left|x_{k, n}\right|}{a_{n}}\right)^{1 / 4} \frac{1}{\left|x-x_{k, n}\right|} \\
& \sim C w(x)^{-1}+\frac{a_{n}^{3 / 2}}{n}\left|p_{n}(x)\right| \sum_{\substack{\left|x_{k, n}\right| \leq a_{\alpha n}, k \neq f j_{x}}}\left(1-\frac{\left|x_{k, n}\right|}{a_{n}}\right)^{3 / 4} \frac{1}{\left|x-x_{k, n}\right|},
\end{aligned}
$$


where we used the fact

$$
1-\frac{\left|x_{k, n}\right|}{a_{2 n}} \sim 1-\frac{\left|x_{k, n}\right|}{a_{n}}, \quad\left|x_{k, n}\right| \leq a_{\alpha n} .
$$

So,

$$
\begin{aligned}
\sum_{n}^{\prime}(x) & \leq C w(x)^{-1}+\frac{a_{n}^{3 / 2}}{n}\left|p_{n}(x)\right| \sum_{\substack{\left|x_{k, n}\right| \leq a_{\alpha n}, k \neq j_{x}}}\left(1-\frac{\left|x_{k, n}\right|}{a_{n}}\right)^{3 / 4} \frac{1}{\left|x_{j_{x}, n}-x_{k, n}\right|} \\
& \leq C w(x)^{-1}+\frac{a_{n}^{3 / 2}}{n}\left|p_{n}(x)\right| \sum_{\substack{\left|x_{k, n}\right| \leq a_{\alpha n}, k \neq j_{x}}}\left(1-\frac{\left|x_{k, n}\right|}{a_{n}}\right)^{3 / 4} \frac{1}{\sum_{j_{x} \lessgtr i \lessgtr k} \varphi_{n}\left(x_{i, n}\right)} \\
& \leq C w(x)^{-1}+a_{n}^{1 / 2}\left|p_{n}(x)\right| \sum_{\substack{\left|x_{k, n}\right| \leq a_{\alpha n}, k \neq j_{x}}}\left(1-\frac{\left|x_{k, n}\right|}{a_{n}}\right)^{3 / 4} \frac{1}{\sum_{j_{x} \lessgtr i \lessgtr k} \sqrt{1-\left|x_{i, n}\right| / a_{n}}} .
\end{aligned}
$$

Therefore we have by Lemma 3.6 with $p=\infty$,

$$
\begin{aligned}
\sum_{n}^{\prime}(x) w(x) \Phi(x)^{3 / 4} \leq & C+C a_{n}^{1 / 2}\left|p_{n}(x)\right| w(x) \Phi(x)^{1 / 4} \\
& \times \sum_{\substack{\left|x_{k, n}\right| \leq a_{\alpha n}, k \neq j_{x}}}\left(1-\frac{\left|x_{k, n}\right|}{a_{n}}\right)^{3 / 4}\left(1-\frac{\left|x_{j_{x}, n}\right|}{a_{n}}\right)^{1 / 2} \frac{1}{\sum_{j_{x} \lessgtr i \lessgtr k} \sqrt{1-\left|x_{i, n}\right| / a_{n}}} \\
\leq & C \sum_{\substack{\left|x_{k, n}\right| \leq a_{\alpha, n}, k \neq j_{x}}} \frac{1}{\left|j_{x}-k\right|} \sim \log n .
\end{aligned}
$$

Lemma 5.3 ([8, Theorem 1]) Let $w \in \mathcal{F}\left(C^{2}+\right)$. Then there exists a constant $C_{0}>0$ such that for every absolutely continuous function $f$ with $w f^{\prime} \in C_{0}(\mathbb{R})$ (this means $w(x) f^{\prime}(x) \rightarrow 0$ as $|x| \rightarrow \infty)$ and every $n \in \mathbb{N}$, we have

$$
E_{n}(w ; f) \leq C \frac{a_{n}}{n} E_{n-1}\left(w ; f^{\prime}\right) .
$$

Proof of Theorem 2.4 There exists $P_{n-1} \in \mathcal{P}_{n}$ such that

$$
\left|\left(f(x)-P_{n-1}(x)\right) w(x)\right| \leq 2 E_{n-1}(w ; f) .
$$

Therefore, by Lemma 5.1 and Lemma 5.2,

$$
\begin{aligned}
& \left|\left(f(x)-L_{n}(f)(x)\right) w(x) \Phi^{3 / 4}(x)\right| \\
& \leq\left|\left(f(x)-P_{n-1}(x)\right) w(x) \Phi^{1 / 4}(x)\right|+\left|L_{n}\left(f-P_{n-1}\right)(x) w(x) \Phi^{3 / 4}(x)\right| \\
& =\left|\left(f(x)-P_{n-1}(x)\right) w(x) \Phi^{3 / 4}(x)\right| \\
& \quad+\left|w(x) \Phi^{3 / 4}(x) \sum_{k=1}^{n}\left(f\left(x_{k, n}\right)-P_{n-1}\left(x_{k, n}\right)\right) w\left(x_{k, n}\right) l_{k, n}(x) w^{-1}\left(x_{k, n}\right)\right|
\end{aligned}
$$




$$
\begin{aligned}
& \leq 2 E_{n-1}(w ; f)\left\{1+w(x) \Phi^{3 / 4}(x)\left|\sum_{k=1}^{n} l_{k, n}(x) w^{-1}\left(x_{k, n}\right)\right|\right\} \\
& \leq C E_{n-1}(w ; f) \log n .
\end{aligned}
$$

Let $w f^{(r)} \in C_{0}(\mathbb{R})$. If we repeatedly use Lemma 5.3, then we have

$$
\left|\left(f(x)-L_{n}(f)(x)\right) w(x) \Phi^{3 / 4}(x)\right| \leq C_{r}\left(\frac{a_{n}}{n}\right)^{r} E_{n-r-1}\left(w ; f^{(r)}\right) \log n .
$$

\section{Competing interests}

The authors declare that they have no competing interests.

\section{Authors' contributions}

All authors conceived of the study, participated in its design and coordination, drafted the manuscript and participated in the sequence alignment. All authors read and approved the final manuscript.

\section{Author details}

'Department of Mathematics Education, Sungkyunkwan University, Seoul, 110-745, Republic of Korea. ${ }^{2}$ Department of Mathematics, Meijo University, Nagoya, 468-8502, Japan.

\section{Acknowledgements}

The authors thank the referees for many kind suggestions and comments.

Received: 10 April 2012 Accepted: 2 October 2012 Published: 17 October 2012

\section{References}

1. Levin, AL, Lubinsky, DS: Orthogonal Polynomials for Exponential Weights. Springer, New York (2001)

2. Jung, HS, Sakai, R: Specific examples of exponential weights. Commun. Korean Math. Soc. 24(2), 303-319 (2009)

3. Damelin, SB, Lubinsky, DS: Necessary and sufficient conditions for mean convergence of Lagrange interpolation for Erdős weights. Can. J. Math. 48(4), 710-736 (1996)

4. Jung, HS, Sakai, R: Derivatives of integrating functions for orthonormal polynomials with exponential-type weights. J. Inequal. Appl. 2009, Article ID 528454 (2009)

5. Jung, HS, Sakai, R: Orthonormal polynomials with exponential-type weights. J. Approx. Theory 152, 215-238 (2008)

6. Mhaskar, HN: Introduction to the Theory of Weighted Polynomial Approximation. World Scientific, Singapore (1996)

7. Lubinsky, DS: A survey of weighted polynomial approximation with exponential weights. Surv. Approx. Theory 3, 1-105 (2007)

8. Sakai, R, Suzuki, N: Favard-type inequalities for exponential weights. Pioneer J. Math. Math. Sci. 3(1), 1-16 (2011)

\section{Submit your manuscript to a SpringerOpen ${ }^{\circ}$ journal and benefit from:}

- Convenient online submission

Rigorous peer review

- Immediate publication on acceptance

- Open access: articles freely available online

- High visibility within the field

- Retaining the copyright to your article 